\title{
Persistent decadal-scale rainfall variability in the tropical South Pacific Convergence Zone through the past six centuries
}

\author{
C. R. Maupin ${ }^{1,2}$, J. W. Partin ${ }^{1}$, C.-C. Shen ${ }^{3}$, T. M. Quinn ${ }^{1,2}$, K. Lin ${ }^{3}$, F. W. Taylor ${ }^{1}$, J. L. Banner ${ }^{2}$, K. Thirumalai ${ }^{1,2}$, \\ and D. J. Sinclair ${ }^{4}$ \\ ${ }^{1}$ Institute for Geophysics, Jackson School of Geosciences, University of Texas, Austin, Texas 78758, USA \\ ${ }^{2}$ Department of Geological Sciences, Jackson School of Geosciences, University of Texas, Austin, Texas 78705, USA \\ ${ }^{3}$ High-Precision Mass Spectrometry and Environment Change Laboratory (HISPEC), Department of Geosciences, \\ National Taiwan University, Taipei 106, Taiwan \\ ${ }^{4}$ Institute of Marine and Coastal Sciences, Rutgers University, New Brunswick, New Jersey 08901, USA
}

Correspondence to: C. R. Maupin (crmaupinatx@neo.tamu.edu)

Received: 25 August 2013 - Published in Clim. Past Discuss.: 10 October 2013

Revised: 23 May 2014 - Accepted: 3 June 2014 - Published: 10 July 2014

\begin{abstract}
Modern Pacific decadal variability (PDV) has global impacts; hence records of PDV from the preinstrumental period are needed to better inform models that are used to project future climate variability. We focus here on reconstructing rainfall in the western tropical Pacific (Solomon Islands; $\sim 9.5^{\circ} \mathrm{S}, \sim 160^{\circ} \mathrm{E}$ ), a region directly influenced by PDV, using cave deposits (stalagmite). A relationship is developed between $\delta^{18} \mathrm{O}$ variations in the stalagmite and local rainfall amount to produce a $600 \mathrm{yr}$ record of rainfall variability from the South Pacific Convergence Zone (SPCZ). We present evidence for large $(\sim 1.5 \mathrm{~m})$, abrupt, and periodic changes in total annual rainfall amount on decadal to multidecadal timescales since $1423 \pm 5 \mathrm{CE}$ (Common Era) in the Solomon Islands. The timing of the decadal changes in rainfall inferred from the 20th-century portion of the stalagmite $\delta^{18} \mathrm{O}$ record coincides with previously identified decadal shifts in PDV-related Pacific ocean-atmosphere behavior (Clement et al., 2011; Deser et al., 2004). The Solomons record of PDV is not associated with variations in external forcings, but rather results from internal climate variability. The $600 \mathrm{yr}$ Solomon Islands stalagmite $\delta^{18} \mathrm{O}$ record indicates that decadal oscillations in rainfall are a persistent characteristic of SPCZ-related climate variability.
\end{abstract}

\section{Introduction}

\subsection{Pacific decadal variability}

Pacific decadal variability (PDV) is intimately linked with global climate variability, and is a critical factor in infrastructure and resource management planning (Cane, 2010; Dong and $\mathrm{Lu}, 2013$; Goddard et al., 2012). Several mechanisms have been proposed to explain the source of the decadalto multidecadal-scale variability in the Pacific, including (1) solar forcing (e.g., Meehl et al., 2009), (2) volcanic forcing (e.g., Crowley, 2000; Robock, 2000), (3) oceanic integration of short-term atmospheric forcing (Clement et al., 2011; Newman et al., 2003), (4) ocean forcing via subtropical cells (Gu and Philander, 1997; McPhaden and Zhang, 2002; Zhang and McPhaden, 2006), and (5) tropical-extratropical feedbacks (Di Lorenzo et al., 2010). There is no consensus, however, on the dynamic mechanism responsible for the decadal variability, which is manifest in a number of atmospheric and oceanic parameters.

A recent example of PDV occurred in 1976/1977, when multiple, basin-scale oceanic and atmospheric parameters shifted coherently to a different mean state, or epoch, that lasted for decades (Mantua and Hare, 2002; Mantua et al., 1997). For example in the ocean, sea-surface temperatures (SST) in the equatorial and eastern tropical Pacific warmed, while the North and South Pacific cooled (Garreaud and 


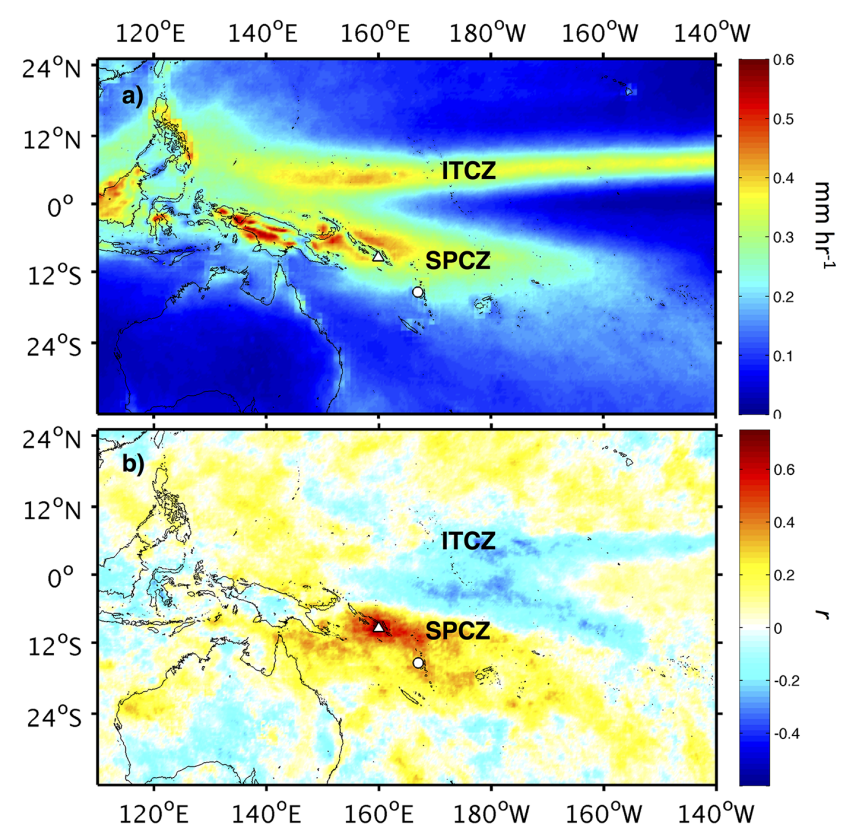

Figure 1. Tropical rainfall patterns in the Indo-Pacific (data from Kummerow et al., 2000). Bands of high rainfall are associated with the Intertropical Convergence Zone (ITCZ) and South Pacific Convergence Zone (SPCZ). (a) Annual mean surface rainfall rate $\left(\mathrm{mm} \mathrm{hr}^{-1}, 1998-2011\right)$. (b) Spatial correlation $(r)$ between monthly rainfall anomalies at Guadalcanal versus other tropical Pacific regions. Anomalies are relative to the 1998-2011 climatology. White triangle denotes the Guadalcanal study site; white circle denotes Espiritu Santo, Vanuatu, a location of a previously published stalagmite record (Partin et al., 2013).

Battisti, 1999; Zhang and McPhaden, 2006). In the atmosphere, sea level pressure (SLP) was higher in the western Pacific and lower in the central and eastern Pacific (L'Heureux et al., 2013; Vecchi et al., 2006), which resulted in reduced trade winds. This SLP pattern is consistent with a weakening of the Pacific Walker circulation (PWC; Dong and $\mathrm{Lu}, 2013$; Vecchi et al., 2006). In addition, wind divergence over the Maritime Continent yielded reduced rainfall in the western tropical Pacific (e.g., Deser et al., 2004; Dong and $\mathrm{Lu}, 2013$ ).

An atmospheric expression of PDV is changes in the position of the South Pacific Convergence Zone (SPCZ, Fig. 1). The SPCZ is the largest persistent rain band in the Southern Hemisphere extending from Papua New Guinea to Tahiti (e.g., Vincent, 1994), making it the primary source of rainfall to Pacific island communities. On decadal timescales, the mean position of the SPCZ moves to the north and east or the south and west depending on the phase of the PDV (e.g., Deser et al., 2004; Dong and Lu, 2013), which has large impacts on local rainfall amount. Two such movements in mean SPCZ position have been identified using instrumental rainfall data (1946-1947, 1976-1977; Deser et al., 2004). Reconstructions of rainfall variability in the pre-instrumental pe-

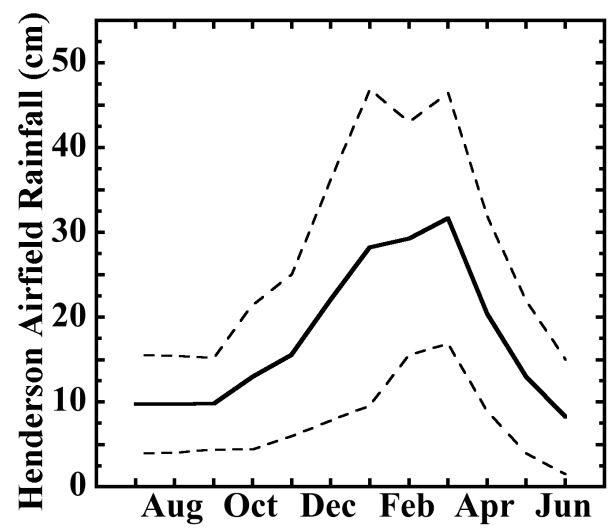

Figure 2. Seasonality of rainfall on northern Guadalcanal. Rain gauge climatology from Henderson Airfield on the northern coast of Guadalcanal (1951-1989), $10 \mathrm{~km}$ northeast of Forestry Cave. Dashed lines indicate $\pm 1 \sigma$ for each climatological month. Rainfall amount over the study site does exhibit strong seasonality, with $65 \%$ of mean total annual rainfall (TAR, $2.10 \pm 0.41 \mathrm{~m}, 1 \sigma, 1952$ 1989) occurring during December through April. Despite this clear seasonality, during the drier season (May through November) rainfall amounts are still considerable.

riod are needed to address whether decadal changes in mean SPCZ position are a persistent characteristic of PDV.

\subsection{Modern climate setting in the Solomon Islands}

The Solomon Islands are positioned within the confluence of SPCZ rainfall (Folland et al., 2002; Vincent, 1994; Widlansky et al., 2010), the rising limb of the PWC and Hadley circulation (Matthews, 2011; Takahashi and Battisti, 2007), and the Western Pacific Warm Pool (WPWP; Rayner et al., 2003). Ocean waters surrounding the Solomon Islands have mean annual SSTs greater than $28^{\circ} \mathrm{C}$, a SST that is often used as a criteria for the boundary of the WPWP (Cravatte et al., 2009; Rayner et al., 2003).

Rainfall variability in the broader tropical SPCZ region is well represented by rainfall variability at the Solomon Islands. Connection of regional variability with local rainfall is accomplished using a short (1998-2011), high-resolution $\left(0.25^{\circ} \times 0.25^{\circ}\right)$ satellite-measured rainfall amount from the Tropical Rainfall Measuring Mission (TRMM; Kummerow et al., 2000). Monthly rainfall anomalies at the Solomon Islands are significantly correlated with rainfall anomalies in the broader tropical SPCZ domain. Rainfall is seasonal at the study site (Fig. 2), with $65 \%$ of mean total annual rainfall (TAR, $2.10 \pm 0.41 \mathrm{~m}, 1 \sigma, 1952-1989)$ occurring during December through April (Vose et al., 1992). These local rainfall observations are based on a monthly rain gauge record from Henderson Airfield, which is located $10 \mathrm{~km}$ northeast of Forestry Cave, on Guadalcanal. We note that, even in May through November, rainfall is still appreciable. 


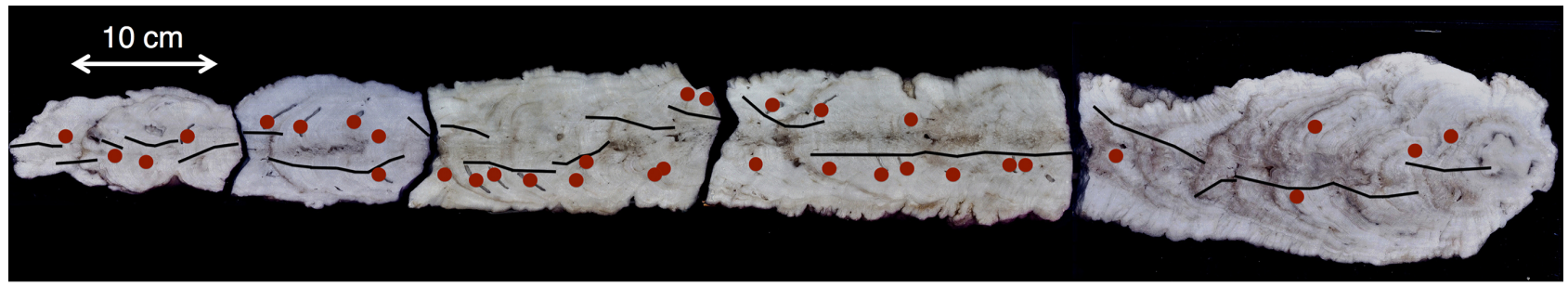

Figure 3. Cross-sectional image of Guadalcanal stalagmite 10FC-02. Stable isotope sample powders were extracted every $500 \mu \mathrm{m}$ along sampling paths shown by black lines. Not all sampling paths are on the same sides of the slabs as depicted. This is to maintain sampling transects on an ideal axis of maximum growth and the center of the growth axis. Red markers on the image indicate sample locations for U-Th dating.

Rainfall varies in the Solomon Islands due to displacement of the SPCZ southwestward or northeastward and/or changes in SPCZ intensity independent of its position (Clement et al., 2011; Deser et al., 2004; Folland et al., 2002). The period from 1977 through 1995 versus 1947 through 1976 had reduced rainfall $(0.5$ mTAR reduction in the Henderson Airfield rain gauge) and reduced cloudiness at the Solomon Islands, which corresponds to a northeastward and equatorward movement of the SPCZ after the 1976/1977 shift. Deconvoluting the effects of movement- and convection-related changes in rainfall amount in the tropical SPCZ requires climate records from multiple locations in the western South Pacific (Deser et al., 2004). The coherent response over the Solomon Islands to rainfall changes triggered by decadal variability make it a well-suited location to begin to reconstruct the decadal signal carried by the tropical portion of the SPCZ.

\subsection{Stalagmite time series from Guadalcanal, Solomon Islands}

Here we present a U-series-dated $\delta^{18} \mathrm{O}$ record of rainfall variability from 2010 to $1423 \pm 5 \mathrm{CE}$ (Common Era) in fastgrowing stalagmites from the northern coast of Guadalcanal, Solomon Islands. We demonstrate that stalagmite $\delta^{18} \mathrm{O}$ variations can be interpreted as variations in rainfall amount through the tropical "amount effect", an empirical relationship whereby greater rainfall amounts lead to more depleted rainfall $\delta^{18} \mathrm{O}$, and thus the $\delta^{18} \mathrm{O}$ of calcite precipitated from this water (Dansgaard, 1964; Gat, 1996; Kurita, 2013; Partin et al., 2012; Rozanski et al., 1993). We quantitatively define the relationship between stalagmite $\delta^{18} \mathrm{O}$ and local rainfall for the modern period and apply this relation over the entire record. This proxy rainfall reconstruction provides a new perspective on tropical SPCZ rainfall variability over the past six centuries, which has important implications for resource management and infrastructure preparation for the Solomon Islands, where fresh water availability is restricted to the volume of a surface lens that is dependent on rainfall amount. It also provides constraints for decadal-range climate model projections.

\section{Methods}

\subsection{Sample collection and description}

Two stalagmites, 10FC-02 and 05FC-04, were collected from Forestry Cave, northwestern Guadalcanal $\left(9^{\circ} 29^{\prime} 19.50^{\prime \prime} \mathrm{S}\right.$, $\left.159^{\circ} 58^{\prime} 25.56^{\prime \prime} \mathrm{E}\right)$. The cave is located $\sim 200 \mathrm{~m}$ a.s.l. on the northern slope of Mount Austen, $2.5 \mathrm{~km}$ south of Barana Village. The slope vegetation consists of dense tropical forest. The cave entrance is positioned on the southwest side of a $\sim 20 \mathrm{~m}$ deep streambed ravine that runs from southeast to northwest. The back of the cave has an elevated $(\sim 2 \mathrm{~m}$ above the cave floor), highly decorated ledge $25-30 \mathrm{~m}$ from the entrance. A rimstone dam forms the face of the ledge. Sample 10FC-02 was collected in August 2010 from this plateau and was in growth position, with an active drip. Sample 05FC-04 used for replication testing (see Sect. 2.4.) is a small "candlestick" stalagmite that was collected broken from the floor of the plateau in November 2005. X-ray diffraction analysis performed on several powdered subsamples of stalagmite10FC-02 show prominent calcite peaks and no aragonite peaks.

Drip waters were collected from both Forestry Cave and nearby Jacob's Cave $\left(9^{\circ} 29^{\prime} 12.42^{\prime \prime} \mathrm{S}, 159^{\circ} 58^{\prime} 23.88^{\prime \prime} \mathrm{E}\right)$ during the same two-week period of August, 2010, when sample 10FC-02 was collected. Although none of the Forestry Cave drips came from directly above sample $10 \mathrm{FC}-02$, they were collected within $3 \mathrm{~m}$ of the sample site. Forestry Cave and Jacob's Cave are $\sim 200 \mathrm{~m}$ apart on the northern slope of Mount Austen and are in the same ravine at the same elevation.

\subsection{Isotopic analyses}

Samples for stable isotopic analysis were continuously milled from stalagmite $10 \mathrm{FC}-02$ at $500 \mu \mathrm{m}$ increments (Fig. 3). This yielded 2140 samples, excluding samples within overlaps used to align different paths. Average temporal resolution of the each sample is $\sim 4$ months (seasonal resolution) of calcite precipitation, based on the average growth rate. Aliquots of each sample, $25-80 \mu \mathrm{g}$, were analyzed for $\delta^{18} \mathrm{O}$ and $\delta^{13} \mathrm{C}$ using a Thermo Electron Kiel IV automated carbonate device connected to a Thermo Electron MAT253 


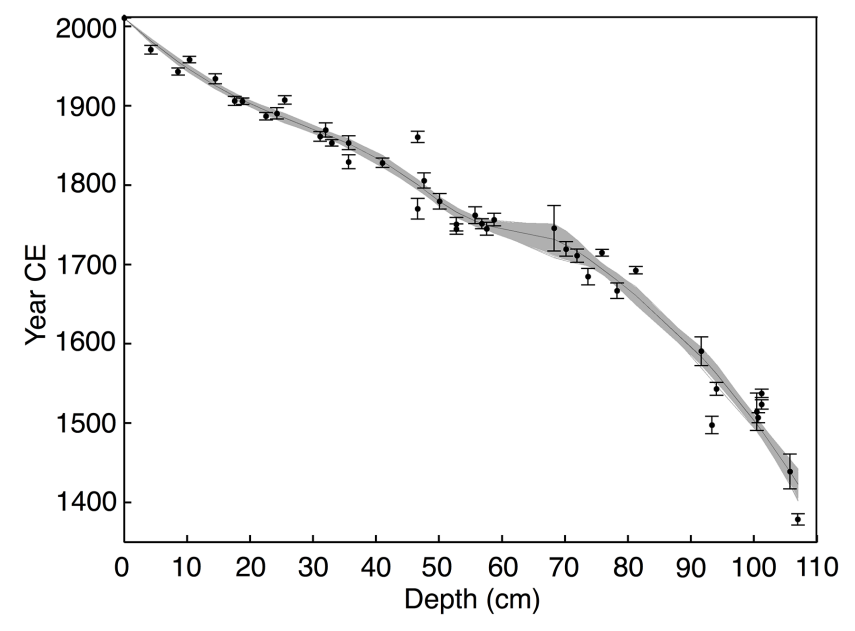

Figure 4. Growth history of Guadalcanal stalagmite 10FC-02. Solid black circles indicate individual U-Th ages with $\pm 2 \sigma$ error bars. The median age model (thin gray line), which assumes no analytical error, and gray cloud, which represents all 10000 alternative age models derived by a Monte Carlo method of least-square fitting of quadratic splines to the U-Th dates and their $\pm 2 \sigma$ age uncertainty, as developed by Partin et al. (2013).

dual-inlet isotope ratio mass spectrometer (IRMS) housed in the Analytical Laboratory for Paleoclimate Studies (ALPS) at the Jackson School of Geosciences, University of Texas at Austin. Stable isotopic ratios of carbonate samples are reported in per mil units versus VPDB (Vienna Pee Dee Belemnite). The long-term average precision for these analyses is $\pm 0.12(2 \sigma)$ for $\delta^{18} \mathrm{O}$ and $\pm 0.06(2 \sigma)$ for $\delta^{13} \mathrm{C}$. Drip water $\delta^{18} \mathrm{O}$ analyses were performed using a Gasbench II attached to a Conflo IV and a Thermo Electron Delta V Plus IRMS, also housed in ALPS. All isotopic ratios for water samples are reported in per mil versus VSMOW (Vienna Standard Mean Ocean Water). Precision for these water analyses is $\pm 0.20(2 \sigma)$ for $\delta^{18} \mathrm{O}$.

$\mathrm{U}-\mathrm{Th}$ dates were determined from stalagmite samples (0.15-1.00 g) on a Thermo Electron Neptune multi-collector inductively coupled plasma mass spectrometer (MC-ICPMS) housed in the High-Precision Mass Spectrometry and Environment Change (HISPEC) Laboratory at the National Taiwan University (Shen et al., 2012). Analyses of stalagmite $10 \mathrm{FC}-02$ samples have an average analytical error of 8.8 years $( \pm 2 \sigma)$.

\subsection{Age models}

The growth history of stalagmite $10 \mathrm{FC}-02$ is constrained by a total of $41 \mathrm{U}-\mathrm{Th}$ dates (Table 1). A Monte Carlo method of constructing alternate age models $(N=10000)$ by leastsquare fitting of quadratic splines to the U-Th dates and their $\pm 2 \sigma$ age uncertainty (Fig. 4; Partin et al., 2013). This method allows construction of an age model that accounts for apparent age inversions of closely spaced U-Th dates, making it particularly useful for generating an age model in a young stalagmite with low $\mathrm{U}$ content. It further provides a method of estimating temporal uncertainty for each individual $\delta^{18} \mathrm{O}$ point in the time series.

We use four U-Th dates and the Monte Carlo method described above to generate the age model for stalagmite 05FC04 . We then align salient features in the $\delta^{18} \mathrm{O}$ record of stalagmite $05 \mathrm{FC}-04$ to the $\delta^{18} \mathrm{O}$ time series of stalagmite 10FC02 , without exceeding the $2 \sigma$ age uncertainties of the U-Th dates.

\subsection{Assessing non-climatic controls on stalagmite $\delta^{18} \mathrm{O}$ variability}

We assess non-climate-related influences on stalagmite $\delta^{18} \mathrm{O}$ using replication and the "Hendy test" (Hendy, 1971). Individual paths in stalagmite 10FC-02 can be offset from the centerline of the stalagmite slabs, and comparing $\delta^{18} \mathrm{O}$ across the stalagmite width comprises one component of a Hendy test. There is no $\delta^{18} \mathrm{O}$ offset between paths that lie toward the margins of the stalagmite versus the center of the stalagmite, indicating that, where sampled, stalagmite $10 \mathrm{FC}-02$ is free of intra-sample non-climatic effects, such as non-equilibrium fractionation and mixed mineralogy. Another component of the Hendy test is the correlation between $\delta^{18} \mathrm{O}$ and $\delta^{13} \mathrm{C}$ variations down the centerline of the stalagmite slab. In the case of stalagmite $10 \mathrm{FC}-02$, the longer of the two samples, the correlation between $\delta^{18} \mathrm{O}$ and $\delta^{13} \mathrm{C}$ is low $\left(R^{2}=0.11\right.$, Fig. 5a, b). We perform a replication test (Dorale and Liu, 2009) by comparing $\delta^{18} \mathrm{O}$ and $\delta^{13} \mathrm{C}$ variations in stalagmites $05 \mathrm{FC}-04$ and $10 \mathrm{FC}-02$. Stalagmite $\delta^{18} \mathrm{O}$ replicates over the 89 years of overlap, in contrast to stalagmite $\delta^{13} \mathrm{C}$. All lines of evidence support a limited role for non-climatic effects on stalagmite $\delta^{18} \mathrm{O}$.

We investigate multiple lines of evidence to assess whether the stalagmite $\delta^{18} \mathrm{O}$ variations record environmental conditions above the cave, which ultimately means the stalagmite reflects changes in rainfall $\delta^{18} \mathrm{O}$. First, we assess whether the calcite $\delta^{18} \mathrm{O}$ formed in isotopic equilibrium with the cave dripwater $\delta^{18} \mathrm{O}$ by calculating the expected equilibrium calcite $\delta^{18} \mathrm{O}$ values using dripwater $\delta^{18} \mathrm{O}$ values and a mean annual temperature for Honiara of $26.5^{\circ} \mathrm{C} \pm 0.1^{\circ} \mathrm{C}$ (as calculated from 1952-1989 meteorological station data from Honiara, GISTEMP, http://data.giss.nasa.gov/gistemp/ station_data/). We use both inorganic, laboratory-based (Kim and O'Neil, 1997) and empirical, cave-based (Tremaine et al., 2011) water-calcite fractionation equations to perform these calculations (Table 2). We acknowledge that the laboratory equation predicts calcite $\delta^{18} \mathrm{O}$ values that are depleted relative to the observed calcite $\delta^{18} \mathrm{O}$. Depletion in cave calcite $\delta^{18} \mathrm{O}$ relative to laboratory equilibrium is well documented (Feng et al., 2012; Tremaine et al., 2011) and has been attributed to differences between typical stalagmite deposition temperatures in cave environments and the temperature ranges of laboratory experiments, as well as stalagmite 
Table 1. U-Th dates used to reconstruct 10FC-02 (top) and 05FC-04 (bottom) stalagmite growth histories.

\begin{tabular}{|c|c|c|c|c|c|c|c|c|c|}
\hline $\begin{array}{l}\text { 10FC-02 } \\
\text { depth }(\mathrm{cm})\end{array}$ & $\begin{array}{l}\text { Weight } \\
\mathrm{g}\end{array}$ & $\begin{array}{l}{ }^{238} \mathrm{U} \\
\mathrm{ppb}\end{array}$ & $\begin{array}{l}{ }^{232} \mathrm{Th} \\
\mathrm{ppt}\end{array}$ & $\begin{array}{l}\delta^{234} \mathrm{U} \\
\text { measured }^{\mathrm{a}}\end{array}$ & $\begin{array}{l}{\left[{ }^{230} \mathrm{Th} /{ }^{238} \mathrm{U}\right]} \\
\text { activity }^{\mathrm{c}}\end{array}$ & $\begin{array}{l}\left.{ }^{230} \mathrm{Th} /{ }^{232} \mathrm{Th}\right] \\
\mathrm{ppm}^{\mathrm{d}}\end{array}$ & $\begin{array}{l}\text { Age } \\
\text { uncorrected }\end{array}$ & $\begin{array}{l}\text { Year } \\
\mathrm{CE}^{\mathrm{c}, \mathrm{e}}\end{array}$ & $\begin{array}{l}\delta^{234} \mathrm{U}_{\text {initial }} \\
\text { corrected }^{\mathrm{b}}\end{array}$ \\
\hline 4.25 & 0.23210 & $204.56 \pm 0.20$ & $44.7 \pm 3.0$ & $6.7 \pm 1.5$ & $0.000432 \pm 0.000044$ & $32.7 \pm 4.0$ & $46.9 \pm 4.8$ & $1970.7 \pm 5.6$ & $6.7 \pm 1.5$ \\
\hline 8.55 & 0.21507 & $295.76 \pm 0.31$ & $55.7 \pm 3.2$ & $9.8 \pm 1.5$ & $0.000679 \pm 0.000035$ & $59.5 \pm 4.6$ & $73.4 \pm 3.8$ & $1943.2 \pm 4.6$ & $9.8 \pm 1.5$ \\
\hline 10.35 & 0.27735 & $333.41 \pm 0.46$ & $46.6 \pm 2.5$ & $6.0 \pm 1.8$ & $0.000528 \pm 0.000032$ & $62.3 \pm 5.1$ & $57.3 \pm 3.5$ & $1958.3 \pm 4.0$ & $6.0 \pm 1.8$ \\
\hline 14.45 & 0.29840 & $213.00 \pm 0.20$ & $18.7 \pm 2.3$ & $5.5 \pm 1.5$ & $0.000738 \pm 0.000056$ & $138 \pm 20$ & $80.2 \pm 6.1$ & $1934.0 \pm 6.2$ & $5.5 \pm 1.5$ \\
\hline 17.55 & 0.21002 & $204.01 \pm 0.21$ & $27.6 \pm 3.3$ & $6.6 \pm 1.5$ & $0.001004 \pm 0.000049$ & $123 \pm 16$ & $109.0 \pm 5.3$ & $1906.2 \pm 5.6$ & $6.6 \pm 1.5$ \\
\hline 18.80 & 0.32690 & $289.38 \pm 0.28$ & $8.6 \pm 2.1$ & $7.0 \pm 1.5$ & $0.000984 \pm 0.000038$ & $544 \pm 136$ & $106.8 \pm 4.1$ & $1905.8 \pm 4.1$ & $7.0 \pm 1.5$ \\
\hline 22.50 & 0.3247 & $193.43 \pm 0.14$ & $12.7 \pm 2.1$ & $6.8 \pm 1.2$ & $0.001164 \pm 0.000042$ & $293 \pm 51$ & $126.4 \pm 4.6$ & $1886.8 \pm 4.7$ & $6.8 \pm 1.2$ \\
\hline 24.25 & 0.16750 & $359.22 \pm 0.41$ & $15.0 \pm 4.2$ & $5.1 \pm 1.9$ & $0.001127 \pm 0.000064$ & $447 \pm 127$ & $122.6 \pm 7.0$ & $1890.4 \pm 7.0$ & $5.1 \pm 1.9$ \\
\hline 25.50 & 22171 & $281.05 \pm 0.31$ & $36.0 \pm 3.1$ & $6.3 \pm 1.7$ & $0.000992 \pm 0.000046$ & $128 \pm 13$ & $107.7 \pm 5.0$ & $1907.3 \pm 5.3$ & $6.3 \pm 1.7$ \\
\hline 31.10 & 0.20897 & $217.47 \pm 0.22$ & $36.8 \pm 3.3$ & $5.0 \pm 1.5$ & $0.001422 \pm 0.000052$ & $139 \pm 14$ & $154.6 \pm 5.7$ & $1861.4 \pm 6.1$ & $5.0 \pm 1.5$ \\
\hline 32.00 & 0.19800 & $212.61 \pm 0.24$ & $38.0 \pm 3.5$ & $9.5 \pm 1.8$ & $0.001358 \pm 0.000079$ & $126 \pm 14$ & $147.0 \pm 8.5$ & $1869.5 \pm 8.8$ & $9.5 \pm 1.8$ \\
\hline 32.95 & 0.3996 & $236.53 \pm 0.21$ & $22.0 \pm 1.8$ & $4.6 \pm 1.2$ & $0.001477 \pm 0.000036$ & $262 \pm 23$ & $160.7 \pm 3.9$ & $1853.2 \pm 4.1$ & $4.6 \pm 1.2$ \\
\hline 35.65 & 0.21855 & $101.31 \pm 0.11$ & $11.1 \pm 3.2$ & $6.2 \pm 1.5$ & $0.001484 \pm 0.000079$ & $224 \pm 65$ & $161.2 \pm 8.6$ & $1853.3 \pm 8.8$ & $6.2 \pm 1.5$ \\
\hline 35.65 & 0.23286 & $152.62 \pm 0.19$ & $29.4 \pm 3.0$ & $5.1 \pm 1.8$ & $0.001723 \pm 0.000077$ & $147 \pm 16$ & $187.3 \pm 8.4$ & $1829.4 \pm 8.8$ & $5.1 \pm 1.8$ \\
\hline 41.00 & 0.23140 & $365.05 \pm 0.40$ & $19.6 \pm 3.0$ & $6.4 \pm 1.6$ & $0.001705 \pm 0.000055$ & $523 \pm 82$ & $185.2 \pm 6.0$ & $1828.1 \pm 6.0$ & $6.4 \pm 1.6$ \\
\hline 46.60 & 0.20286 & $216.07 \pm 0.26$ & $50.1 \pm 3.4$ & $9.0 \pm 1.8$ & $0.001452 \pm 0.000059$ & $103.3 \pm 8.2$ & $157.3 \pm 6.4$ & $1770.1 \pm 7.1$ & $9.0 \pm 1.8$ \\
\hline 46.60 & 0.27950 & $182.66 \pm 0.22$ & $11.3 \pm 2.5$ & $10.8 \pm 1.9$ & $0.00225 \pm 0.00012$ & $602 \pm 137$ & $243 \pm 13$ & $1861 \pm 13$ & $10.8 \pm 1.9$ \\
\hline 47.60 & 0.20568 & $146.86 \pm 0.12$ & $40.2 \pm 3.4$ & $8.2 \pm 1.4$ & $0.001968 \pm 0.000083$ & $119 \pm 11$ & $213.3 \pm 9.0$ & $1805.8 \pm 9.7$ & $8.3 \pm 1.4$ \\
\hline 50.10 & 0.3801 & $240.37 \pm 0.31$ & $9.9 \pm 1.8$ & $4.8 \pm 1.7$ & $0.002141 \pm 0.000089$ & $861 \pm 164$ & $232.9 \pm 9.7$ & $1779.6 \pm 9.7$ & $4.8 \pm 1.7$ \\
\hline 52.75 & 0.19978 & $161.95 \pm 0.19$ & $13.2 \pm 3.5$ & $6.6 \pm 1.7$ & $0.002423 \pm 0.000077$ & $492 \pm 131$ & $263.1 \pm 8.4$ & $1744.4 \pm 8.5$ & $6.6 \pm 1.7$ \\
\hline 52.75 & 0.3447 & $223.14 \pm 0.32$ & $22.9 \pm 2.0$ & $5.6 \pm 1.8$ & $0.002481 \pm 0.000059$ & $399 \pm 36$ & $269.7 \pm 6.4$ & $1750.6 \pm 6.6$ & $5.6 \pm 1.8$ \\
\hline 55.70 & 0.18103 & $172.38 \pm 0.18$ & $35.4 \pm 3.8$ & $7.4 \pm 1.8$ & $0.002351 \pm 0.000095$ & $189 \pm 22$ & $255 \pm 10$ & $1762 \pm 11$ & $7.4 \pm 1.8$ \\
\hline 56.80 & 0.2442 & $245.80 \pm 0.55$ & $30.8 \pm 2.9$ & $3.2 \pm 2.9$ & $0.002417 \pm 0.000057$ & $318 \pm 30$ & $263.4 \pm 6.2$ & $1751.3 \pm 6.4$ & $3.2 \pm 2.9$ \\
\hline 57.55 & 23711 & $142.07 \pm 0.13$ & $26.6 \pm 2.9$ & $6.5 \pm 1.3$ & $0.002501 \pm 0.000071$ & $221 \pm 25$ & $271.7 \pm 7.8$ & $1744.9 \pm 8.2$ & $6.5 \pm 1.3$ \\
\hline 58.75 & 21828 & $156.06 \pm 0.16$ & $23.3 \pm 3.2$ & $6.7 \pm 1.5$ & $0.002385 \pm 0.000071$ & $264 \pm 37$ & $259.1 \pm 7.8$ & $1756.5 \pm 8.0$ & $6.7 \pm 1.5$ \\
\hline 68.25 & 0.21947 & $124.52 \pm 0.16$ & $26.3 \pm 3.2$ & $7.2 \pm 2.0$ & $0.00250 \pm 0.00026$ & $196 \pm 31$ & $272 \pm 28$ & $1746 \pm 29$ & $7.2 \pm 2.0$ \\
\hline 70.15 & 0.2322 & $148.03 \pm 0.20$ & $21.1 \pm 3.0$ & $6.8 \pm 1.9$ & $0.00272 \pm 0.00008$ & $316 \pm 46$ & $295.8 \pm 9.0$ & $1719.4 \pm 9.2$ & $6.8 \pm 1.9$ \\
\hline 71.90 & 0.30160 & $227.11 \pm 0.24$ & $7.7 \pm 2.3$ & $7.8 \pm 1.8$ & $0.002781 \pm 0.000078$ & $1347 \pm 403$ & $301.8 \pm 8.5$ & $1711.0 \pm 8.5$ & $7.8 \pm 1.8$ \\
\hline 73.65 & 0.3151 & $128.17 \pm 0.22$ & $16.0 \pm 2.2$ & $6.0 \pm 2.3$ & $0.003036 \pm 0.000094$ & $403 \pm 57$ & $330 \pm 10$ & $1685 \pm 10$ & $6.0 \pm 2.3$ \\
\hline 78.25 & 0.15010 & $243.57 \pm 0.31$ & $22.3 \pm 4.6$ & $8.5 \pm 2.2$ & $0.003204 \pm 0.000089$ & $577 \pm 121$ & $347.5 \pm 9.7$ & $1714.8 \pm 9.8$ & $8.5 \pm 2.2$ \\
\hline 91.65 & 0.21409 & $115.82 \pm 0.15$ & $32.8 \pm 3.3$ & $7.8 \pm 2.1$ & $0.00395 \pm 0.00016$ & $230 \pm 25$ & $429 \pm 18$ & $1667 \pm 18$ & $7.8 \pm 2.1$ \\
\hline 93.30 & 0.21070 & $176.42 \pm 0.23$ & $38.6 \pm 3.3$ & $5.9 \pm 1.7$ & $0.004779 \pm 0.000094$ & $361 \pm 32$ & $520 \pm 10$ & $1692.7 \pm 11$ & $5.9 \pm 1.7$ \\
\hline 100.5 & 0.21616 & $122.5 \pm 0.12$ & $33.4 \pm 3.2$ & $7.9 \pm 1.7$ & $0.00465 \pm 0.00021$ & $281 \pm 30$ & $505 \pm 23$ & $1591 \pm 24$ & $8.0 \pm 1.7$ \\
\hline 105.70 & 0.1656 & $140.70 \pm 0.18$ & $17.3 \pm 4.2$ & $7.5 \pm 1.9$ & $0.00530 \pm 0.00020$ & $710 \pm 174$ & $576 \pm 22$ & $1498 \pm 22$ & $7.5 \pm 1.9$ \\
\hline 75.88 & 0.85206 & $121.08 \pm 0.11$ & $11.15 \pm 0.82$ & $4.2 \pm 1.4$ & $0.002757 \pm 0.000037$ & $494 \pm 37$ & $300.2 \pm 4.1$ & $1543.1 \pm 4.3$ & $4.3 \pm 1.4$ \\
\hline 81.23 & 1.00879 & $126.18 \pm 0.11$ & $6.85 \pm 0.69$ & $3.7 \pm 1.4$ & $0.002950 \pm 0.000028$ & $897 \pm 91$ & $321.4 \pm 3.1$ & $1514.4 \pm 3.2$ & $3.7 \pm 1.4$ \\
\hline 94.03 & 0.6034 & $143.33 \pm 0.13$ & $8.9 \pm 1.2$ & $4.2 \pm 1.4$ & $0.004323 \pm 0.000075$ & $1143 \pm 149$ & $471.1 \pm 8.3$ & $1506.9 \pm 8.3$ & $4.2 \pm 1.4$ \\
\hline 101.23 & 0.40424 & $140.12 \pm 0.14$ & $15.4 \pm 1.7$ & $4.5 \pm 1.6$ & $0.004516 \pm 0.000051$ & $680 \pm 77$ & $492.1 \pm 5.7$ & $1523.5 \pm 5.9$ & $4.5 \pm 1.6$ \\
\hline 100.68 & 0.6377 & $136.11 \pm 0.21$ & $10.7 \pm 1.1$ & $2.7 \pm 1.9$ & $0.004652 \pm 0.000056$ & $979 \pm 101$ & $507.8 \pm 6.2$ & $1537.6 \pm 6.3$ & $2.7 \pm 1.9$ \\
\hline 101.23 & 0.66251 & $144.06 \pm 0.24$ & $8.2 \pm 1.1$ & $2.2 \pm 2.3$ & $0.004364 \pm 0.000046$ & $1271 \pm 164$ & $476.6 \pm 5.2$ & $1438.8 \pm 5.2$ & $2.2 \pm 2.3$ \\
\hline 106.98 & 0.52911 & $112.38 \pm 0.13$ & $23.5 \pm 1.3$ & $4.3 \pm 1.8$ & $0.005865 \pm 0.000058$ & $463 \pm 26$ & $639.6 \pm 6.4$ & $1378.5 \pm 7.0$ & $4.3 \pm 1.8$ \\
\hline $\begin{array}{l}\text { 05FC-04 } \\
\text { depth }(\mathrm{cm})\end{array}$ & $\begin{array}{l}\text { Weight } \\
\mathrm{g}\end{array}$ & $\begin{array}{l}{ }^{238} \mathrm{U} \\
\mathrm{ppb}\end{array}$ & $\begin{array}{l}{ }^{232} \mathrm{Th} \\
\mathrm{ppt}\end{array}$ & $\begin{array}{l}\delta^{234} \mathrm{U} \\
\text { measured }^{\mathrm{a}}\end{array}$ & $\begin{array}{l}{\left[{ }^{230} \mathrm{Th} /{ }^{238} \mathrm{U}\right]} \\
\text { activity }\end{array}$ & $\begin{array}{l}{\left[{ }^{230} \mathrm{Th} /{ }^{232} \mathrm{Th}\right]} \\
\mathrm{ppm}^{\mathrm{d}}\end{array}$ & $\begin{array}{l}\text { Age } \\
\text { uncorrected }\end{array}$ & $\begin{array}{l}\text { Year } \\
C^{c, e}\end{array}$ & $\begin{array}{l}\delta^{234} \mathrm{U}_{\text {initial }} \\
\text { corrected }^{\mathrm{b}}\end{array}$ \\
\hline 0.020 & 0.20850 & $354.39 \pm 0.29$ & $14.0 \pm 3.3$ & $5.3 \pm 1.1$ & $0.000345 \pm 0.000039$ & $145 \pm 38$ & $37.5 \pm 4.2$ & $1975.5 \pm 4.2$ & $5.3 \pm 1.1$ \\
\hline 4.665 & 0.38044 & $232.36 \pm 0.29$ & $19.6 \pm 1.8$ & $4.8 \pm 1.9$ & $0.000732 \pm 0.000043$ & $144 \pm 16$ & $79.6 \pm 4.6$ & $1934.6 \pm 4.8$ & $4.8 \pm 1.9$ \\
\hline 12.765 & 0.36680 & $226.79 \pm 0.19$ & $16.7 \pm 1.9$ & $4.4 \pm 1.2$ & $0.001142 \pm 0.000044$ & $256 \pm 31$ & $124.2 \pm 4.8$ & $1889.7 \pm 4.9$ & $4.4 \pm 1.2$ \\
\hline 16.755 & 0.33310 & $217.71 \pm 0.21$ & $9.9 \pm 2.1$ & $7.1 \pm 1.5$ & $0.001224 \pm 0.000094$ & $443 \pm 99$ & $133 \pm 10$ & $1880 \pm 10$ & $7.1 \pm 1.5$ \\
\hline
\end{tabular}

Analytical errors are $2 \sigma$ of the mean.

a $\delta^{234} \mathrm{U}=\left(\left[{ }^{234} \mathrm{U} /{ }^{238} \mathrm{U}\right]_{\text {activity }}-1\right) \times 1000$.

b $\delta^{234} \mathrm{U}_{\text {initial }}$ corrected was calculated based on ${ }^{230} \mathrm{Th}$ age (T), i.e., $\delta^{234} \mathrm{U}_{\text {initial }}=\delta^{234} \mathrm{U}_{\text {measured }} X \mathrm{e}^{\lambda 234 * T}$, and $T$ is corrected age.

${ }^{\mathrm{c}}\left[{ }^{230} \mathrm{Th} /{ }^{238} \mathrm{U}\right]_{\text {activity }}=1-\mathrm{e}^{-\lambda 230 T}+\left(\delta^{234} \mathrm{U}_{\text {measured }} / 1000\right)\left[\lambda_{230} /\left(\lambda_{230}-\lambda_{234}\right)\right]\left(1-\mathrm{e}^{-(\lambda 230-\lambda 234) T}\right)$, where $T$ is the age.

Decay constants are $9.1577 \times 10^{-6} \mathrm{yr}^{-1}$ for ${ }^{230} \mathrm{Th}, 2.8263 \times 10^{-6} \mathrm{yr}^{-1}$ for ${ }^{234} \mathrm{U}$, and $1.55125 \times 10^{-10} \mathrm{yr}^{-1}$ for ${ }^{238} \mathrm{U}$ (Cheng et al., 2000; Jaffey et al., 1971).

d The degree of detrital ${ }^{230} \mathrm{Th}$ contamination is indicated by the $\left[{ }^{230} \mathrm{Th} /{ }^{232} \mathrm{Th}\right]$ atomic ratio instead of the activity ratio.

${ }^{\mathrm{e}}$ Age corrections were calculated using an estimated atomic ${ }^{230} \mathrm{Th} /{ }^{232} \mathrm{Th}$ ratio of $4 \pm 2 \mathrm{ppm}$.

growth rate (Feng et al., 2012; Mickler et al., 2006). The $\delta^{18} \mathrm{O}$ value predicted from the cave-based fractionation equation of Tremaine et al. (2011) falls within error of the calcite $\delta^{18} \mathrm{O}$ measured at the tip of stalagmite $10 \mathrm{FC}-02$, a sample that includes the top $500 \mu \mathrm{m}$ of the stalagmite $10 \mathrm{FC}-02$ and which may represent an average value for at least the preceding $\sim 3$ months. Next we assess whether or not calcite $\delta^{18} \mathrm{O}$, which reflects cave dripwater $\delta^{18} \mathrm{O}$, is in fact recording rainfall $\delta^{18} \mathrm{O}$ changes above the cave. The $\delta^{18} \mathrm{O}$ signal of stalagmite $05 \mathrm{FC}-04$ closely matches the $\delta^{18} \mathrm{O}$ signal in 
Table 2. Summary of dripwater, predicted and actual calcite $\delta^{18} \mathrm{O}$ values. Predicted values were calculated using drip water $\delta^{18} \mathrm{O}$ values; experimental, laboratory-based as well as cave-based, empirical dripwater-calcite fractionation equations; and mean annual temperature for Honiara from 1952 to $1989\left(26.5^{\circ} \mathrm{C} \pm 0.1^{\circ} \mathrm{C}, 1 \mathrm{se}\right)$ from GISTEMP station data. Forestry Cave and Jacob's Cave are $\sim 200 \mathrm{~m}$ apart on the northern slope of Mount Austen. The tip $\delta^{18} \mathrm{O}$ value is the measured value for the top $500 \mu \mathrm{m}$ of the $10 \mathrm{FC}-02$ stalagmite and represents an average value for the preceding $\sim 4$ months. Predicted and measured values of $\delta^{18} \mathrm{O}$ calcite fall within error of each other using the cave-based equation, even when only accounting for analytical uncertainty.

\begin{tabular}{|c|c|c|c|c|}
\hline & & $26.5^{\circ} \mathrm{C}$ & & \\
\hline & $\begin{array}{l}\text { Forestry } \\
\text { Cave drip }\end{array}$ & $\begin{array}{l}\text { Jacob's Cave } \\
\text { drip } 1\end{array}$ & $\begin{array}{l}\text { Jacob's Cave } \\
\text { drip } 2\end{array}$ & $\begin{array}{l}2 \sigma \\
\text { uncertainty }\end{array}$ \\
\hline Measured $\delta^{18} \mathrm{O}(\% \circ \mathrm{VSMOW})$ & -6.79 & -6.85 & -6.60 & \pm 0.20 \\
\hline Kim and O'Neil calcite (lab) $\delta^{18} \mathrm{O}(\% \circ$ VPDB $)$ & -9.17 & -9.23 & -8.98 & \pm 0.20 \\
\hline \multirow[t]{3}{*}{ Tremaine calcite (cave) $\delta^{18} \mathrm{O}(\% \circ \mathrm{VPDB})$} & -8.05 & -8.11 & -7.86 & \pm 0.20 \\
\hline & & & $\begin{array}{l}\text { 10FC-02 tip } \\
\delta^{18} \mathrm{O} \\
(\% \circ \mathrm{VPDB})\end{array}$ & $\begin{array}{l}2 \sigma \\
\text { uncertainty }\end{array}$ \\
\hline & & & -7.59 & \pm 0.12 \\
\hline
\end{tabular}

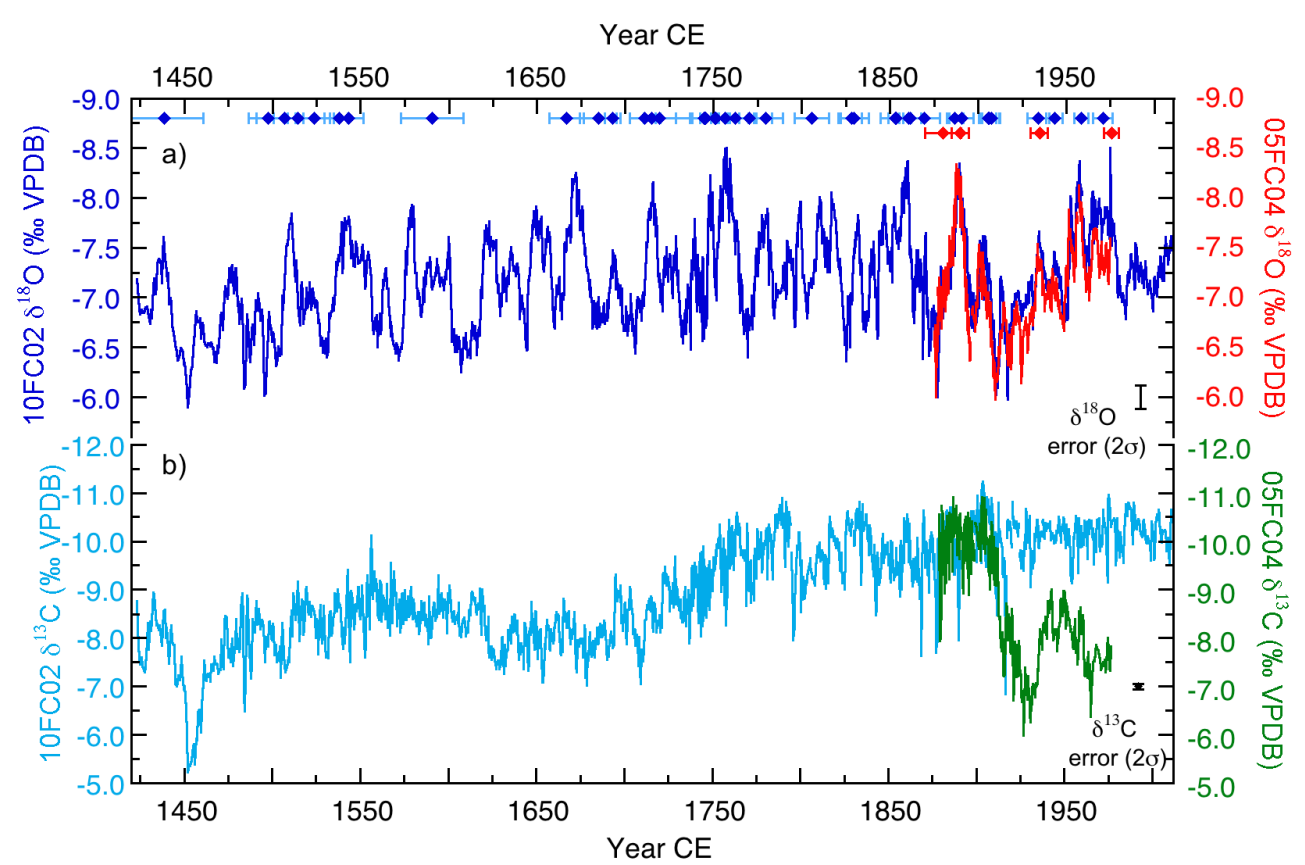

Figure 5. Stable oxygen isotopic variations in Guadalcanal stalagmites $10 \mathrm{FC}-02$ and $05 \mathrm{FC}-04$. Blue (red) diamonds with $\pm 2 \sigma$ error bars illustrate the locations of 10FC-02 (05FC-04) U-Th ages; black circles with $\pm 2 \sigma$ errors denote uncertainties for stable isotopic measurements. There is strong reproducibility in the $\delta^{18} \mathrm{O}$ in the two stalagmites (panel a), in contrast to the limited reproducibility in the $\delta^{13} \mathrm{C}$ records (panel b). There is a weak correlation $\left(R^{2}=0.11, p<0.01\right)$ between the oxygen and carbon isotope records in the $10 \mathrm{FC}-02$ stalagmite. The chronology of the $05 \mathrm{FC}-04$ stalagmite record has been adjusted by $\sim 5$ years to maximize the goodness of fit between the $\delta^{18} \mathrm{O}$ records. This adjustment is less than the \pm 6.0 -year average uncertainty for the $05 F C-02$ stalagmite U-Th dates.

stalagmite10FC-02: a demonstration of the reproducibility of the climate signal (Fig. 5a). Stalagmite $\delta^{13} \mathrm{C}$ variations show none of the decadal variability exhibited by the stalagmite $\delta^{18} \mathrm{O}$ record. The controls on stalagmite $\delta^{13} \mathrm{C}$ are more complicated, and the contrast of the disagreement between the two stalagmites $\delta^{13} \mathrm{C}$ records and the agreement between the two $\delta^{18} \mathrm{O}$ records further increases our confidence that stalagmite $\delta^{18} \mathrm{O}$ is recording a common signal: rainfall $\delta^{18} \mathrm{O}$. We conclude from the above analyses that the stalagmite $\delta^{18} \mathrm{O}$ best reflects rainfall $\delta^{18} \mathrm{O}$ composition over the stalagmite growth interval. 


\section{Results and implications}

\subsection{Controls on modern $\delta^{18} \mathrm{O}$}

\subsubsection{Controls on modern rainfall $\delta^{18} \mathrm{O}$ on tropical iuslands}

Rainfall $\delta^{18} \mathrm{O}$ on tropical islands is controlled by the scale of the convective system, rather than the source of the water vapor (Kurita, 2013), a finding that indicates that mesoscale convection plays a key role in the amount effect (e.g., Rozanski et al., 1993; Gat, 1996). Mesoscale SPCZ convection is the primary source of wet-season rainfall for Forestry Cave, suggesting that this convection type dominates the isotopic composition of rainfall and by inference the stalagmite record. We further investigate how moisture source effects may complicate the relationship between rainfall amount and rainfall $\delta^{18} \mathrm{O}$ by examining the potential pathways and sources of water vapor to the Solomon Islands. We use model results from mid-month of the peak dry season (August and September) and mid-month of the peak wet season (February and March) from 2007 to 2009 (HYSPLIT, http://www.arl.noaa.gov/HYSPLIT.php). The resulting trajectories show possible air parcel paths and potential vapor sources ranging from the equatorial Pacific to the eastern tropical and subtropical South Pacific. These trajectories represent a broad range of potential water vapor sources, transit distances, and mean and seasonal SST regimes, all over an interval of time where stalagmite $\delta^{18} \mathrm{O}$ changes by only $0.3 \%$. This evidence suggests that rainfall source is not an important factor in controlling rainfall $\delta^{18} \mathrm{O}$ in the modern setting of the SPCZ.

\subsubsection{Relationship between stalagmite $\delta^{18} \mathrm{O}$ and rainfall amount}

We compare stalagmite $\delta^{18} \mathrm{O}$ with local rain gauge observations (Fig. 6) at two locations (Henderson Airfield on Guadalcanal; Auki, on Malaita) to create a transfer function between the two variables. It would be ideal to have contemporaneous rainfall amount, rainfall $\delta^{18} \mathrm{O}$, cave dripwater $\delta^{18} \mathrm{O}$, and stalagmite $\delta^{18} \mathrm{O}$ time series. However, as we do not have time series of rainfall $\delta^{18} \mathrm{O}$ or dripwater $\delta^{18} \mathrm{O}$, we establish in Sect. 2.4 that stalagmite $\delta^{18} \mathrm{O}$ records change in rainfall $\delta^{18} \mathrm{O}$. Therefore, we use this relationship to calibrate the changes in stalagmite $\delta^{18} \mathrm{O}$ (i.e., rainfall $\delta^{18} \mathrm{O}$ ) to rainfall amount. We calculate the dependence of stalagmite $\delta^{18} \mathrm{O}$ changes on changes in total annual rainfall amount through robust maximum likelihood linear regression (e.g., Thirumalai et al., 2011) of means and corresponding standard errors from both rain gauge records against contemporaneous means and standard errors in the stalagmite $\delta^{18} \mathrm{O}$ record across known regime shifts in the PDV during which local rainfall data exist. We take this approach because it relates rainfall amount to stalagmite $\delta^{18} \mathrm{O}$

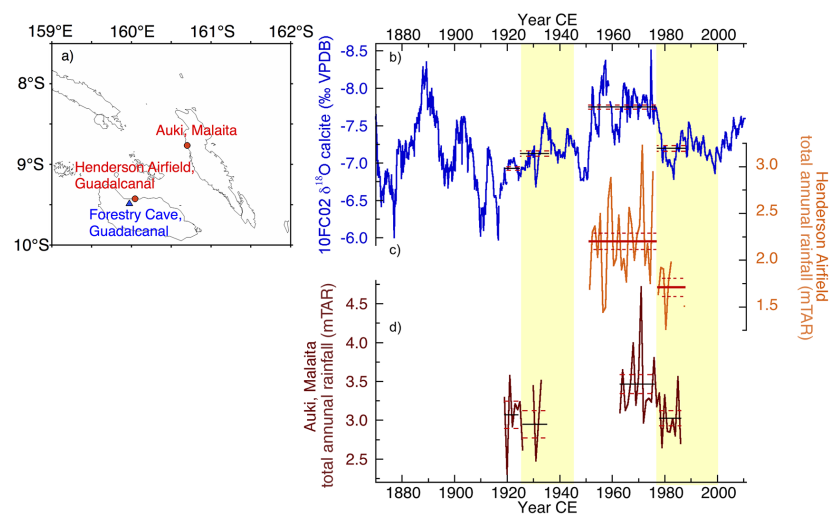

Figure 6. Comparison between stalagmite $\delta^{18} \mathrm{O}$ and local rainfall records. (a) Location map of Forestry Cave (blue triangle) and rain gauge data sites (red circles: Henderson Airfield, Honiara, Guadalcanal; Auki, Malaita, Solomon Islands). (b) $\delta^{18} \mathrm{O}$ variations in stalagmite 10FC-02 from 1870 to 2009 . Total annual rainfall (TAR; data from Deser et al. (2004) and Vose et al. (1992)) at (c) Henderson Airfield and (d) Auki. Mean (solid horizontal lines) and standard error (dashed horizontal lines) of TAR values associated with select epoch intervals of PDV are also shown. Beige stippled (white) area highlights positive (negative) phases of the Interdecadal Pacific Oscillation (IPO; Folland et al., 2002).

across phase changes in PDV (1946/1947; 1976/1977). The relationship between mean total annual rainfall (mTAR) and stalagmite $\delta^{18} \mathrm{O}$ is $1.4 \pm 0.3 \%$ o TAR $^{-1}(r=-0.92, n=6$; Fig. 7). Annual rainfall totals were calculated, as opposed to means or anomalies, for purposes of comparison to the stalagmite record. Annual totals were only calculated for years where gauge observations exist for all 12 months of the year. A similar stalagmite study from Vanuatu derived a relationship of $1 \%$ oper $1.4 \mathrm{~m}$ rainfall/wet season (Partin et al., 2013). These results are consistent with the relationship between rainfall amount and rainfall $\delta^{18} \mathrm{O}$ of $1.2 \%$ o $\mathrm{mTAR}^{-1}$ observed at the closest available Global Network of Isotopes in Precipitation (GNIP) site in Madang, Papua New Guinea, $1650 \mathrm{~km}$ to the west-northwest of Guadalcanal. Rainfall $\delta^{18} \mathrm{O}$ and rainfall amount from Guam have a similar relationship of $1.18 \pm 0.31 \%$ mTAR $^{-1}$ (Partin et al., 2012). The spatial and temporal consistency of the stalagmite $\delta^{18} \mathrm{O}$ amount effect relationship in the western tropical Pacific over the past 100 years suggests that this relationship can be used in the Solomons over the past 600 years.

\subsection{Characterizing the rainfall reconstruction}

Our stalagmite $\delta^{18} \mathrm{O}-$ rainfall amount relationship (Fig. 7) reveals that rainfall variability in the Solomon Islands over the past six centuries is characterized by large, abrupt, periodic changes in rainfall amount on decadal to multidecadal timescales. The well-studied transition in 1976/1977 of Pacific climate represents a relatively small change when compared to many such shifts observed throughout the 


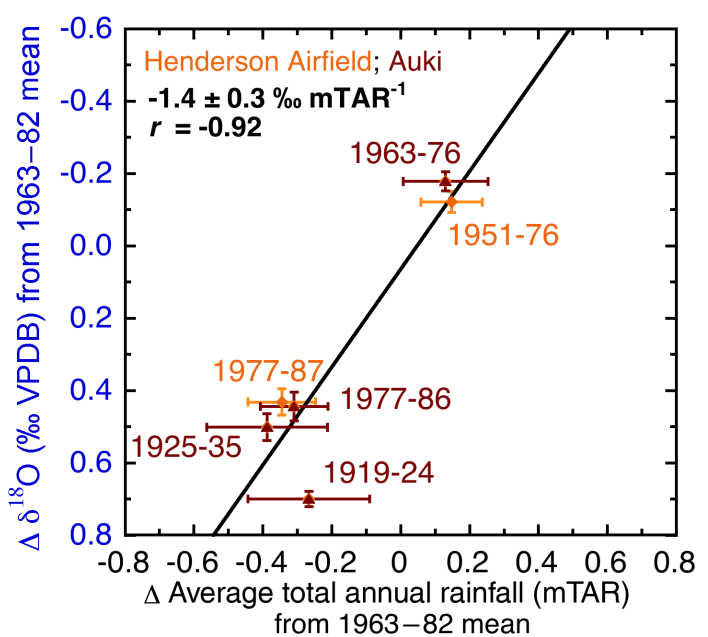

Figure 7. Plot of stalagmite $\delta^{18} \mathrm{O}$ versus total annual rainfall from local rain gauge data. Means and standard errors were calculated for rain gauge and stalagmite $\delta^{18} \mathrm{O}$ records during contemporaneous intervals within individual known PDV phases, when data exist for both time series. Labels indicate the interval over which means and standard errors were calculated. Means for each of the three respective time series over the only time interval in which all three overlap, 1963-1982, were removed from respective values in order to standardize the cross plot to account for the difference in total rainfall amount at Auki and Henderson Airfield.

Solomon Islands rainfall reconstruction. For example, the pre-instrumental portion of the stalagmite $\delta^{18} \mathrm{O}$ record contains decadal transitions that routinely reach or exceed 1.5 mTAR $(\sim 2.4 \%$ ). Such values are three times as large as the wet-to-dry transition of 0.5 mTAR $(\sim 0.6 \%)$ that occurred in 1976/1977. Transitions from periods of high to low rainfall (and vice versa) evolve abruptly ( $<20$ years) every 12 to 62 years.

Multiple spectral analysis techniques (wavelet; Fig. 8, Monte Carlo-singular spectrum analysis (MC-SSA) and the multi-taper method (MTM; Fig. 9; Liu et al., 2007; Torrence and Compo, 1998) all independently confirm the oscillatory nature of the proxy rainfall record. MTM and wavelet analyses reveal persistent concentrations of power at decadal periodicities, significant above an $\mathrm{AR}(1)$ red noise model ( $p<0.05$ for the wavelet and $p<0.01$ in the MTM). SSA demonstrates that $86 \%$ of the variance in the stalagmite record (1423-2010 CE) is decadal to multidecadal in nature (10 to 50 years). The wavelet spectrum also shows significant power in the annual to interannual band, when stalagmite growth is rapid enough.

\subsection{Multi-century replication of SPCZ variability}

We compare the stalagmite record from the Solomon Islands to a stalagmite record from nearby Vanuatu over the common interval of stalagmite growth (1557-2003 CE) to test the regional coherence of the reconstructed rainfall variability associated with the SPCZ (Fig. 10; Partin et al., 2013). The proxy rainfall record of Partin et al. (2013), documented decadal to multidecadal-scale rainfall changes within the SPCZ, $\sim 1000 \mathrm{~km}$ to the southeast of the Solomon Islands study site. This proxy rainfall variability at Vanuatu was attributed to internal climate variability associated with the SPCZ, and not to external forcing. SSA analysis reveals that the first three principal components (PCs) are the same in both records $(\sim 52 \mathrm{yr}, \sim 28 \mathrm{yr}, \sim 16 \mathrm{yr}$; the average period of each PC in the two records, Fig. 10) and account for over $70 \%$ of the variance in each. The percent variance explained by each PC is $40 \%$ at $\sim 52$ years, $25 \%$ at $\sim 28$ years, and $10 \%$ at $\sim 16$ years. The SSA results confirm a common signal at decadal to multidecadal timescales, which likely derives from regional-scale changes in the SPCZ. We note that MTM results identify concentrations of variance exceeding the $95 \%$ confidence limit between 10 and 20 years in the Solomons stalagmite record, but not in the Vanuatu stalagmite record (Partin et al., 2013, Fig. 2b).

Decadal-scale variability is captured in coral proxy records from the SPCZ region that are more than a century long. SPCZ variability is manifest as changes in seasurface salinity (SSS) and SST at Fiji (Linsley et al., 2006; 2008; Wu et al., 2013) and Vanuatu (Gorman et al., 2012). SSS variability at Fiji and Vanuatu reflects a combination of water mass advection and local balance of precipitationevaporation. Thus, the coral proxy primarily reflects surfaceocean variability, which is not necessarily the atmospheric phenomenon that drives rainfall variability.

\subsection{Stalagmite variability: external forcing vs. internal variability}

The demonstration that there is a coherent signal in SPCZ rainfall variability over the past several centuries in the stalagmite records from the Solomon Islands and Vanuatu allows us to assess several mechanisms that have been proposed to drive climate variability in this region. Solar, volcanic, and anthropogenic forcing are three external forcings that have the potential to drive the variability observed in the stalagmite records from the SPCZ.

The $600 \mathrm{yr}$ stalagmite record from the Solomon Islands can be used to assess the potential impacts of changes in total solar irradiation on SPCZ variability (Fig. 11). On century scales, there is no persistent or consistent relationship between stalagmite $\delta^{18} \mathrm{O}$ variability and the solar variability reconstruction of Vieira et al. (2011). Periods of high solar irradiance (e.g., solar maxima centered at $1600 \mathrm{CE}$ ) and low solar irradiance (e.g., Maunder Minimum centered at 


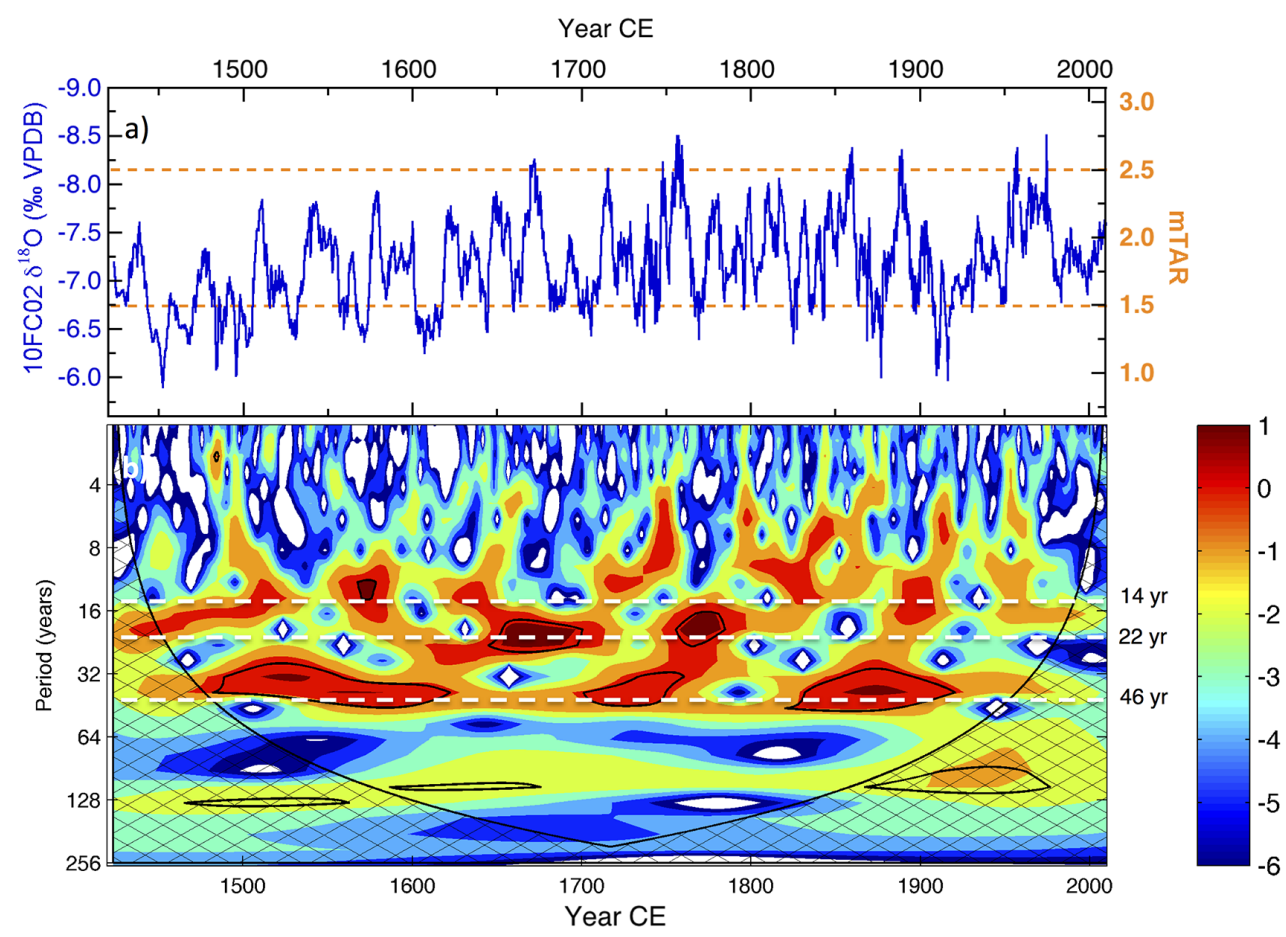

Figure 8. (a) Variations in stalagmite $\delta^{18} \mathrm{O}$ (left axis) and calculated rainfall (mTAR; right axis) as derived from the relationship in Fig. 6. Horizontal dashed orange lines highlight a change of 1 mTAR that approximates the average range of multidecadal rainfall variability in the record. (b) Wavelet spectrum of stalagmite $\delta^{18} \mathrm{O}$ time series (Liu et al., 2007; Torrence and Compo, 1998). Black contours denote power above the $95 \%$ confidence interval when using an AR(1) red noise model; crosshatching represents the "cone of influence" (Torrence and Compo, 1998). The horizontal dashed white lines denote the period of the first 3 principal components (PCs) of the stalagmite record as calculated using singular spectral analysis and 10 components with a window length of 50. The first PC is centered at 46 years and explains $44 \%$ of the variance; the second PC is centered at 22 years and explains $23 \%$ of the variance; while the third PC is centered at 14 years and explains $13 \%$ of the variance.

$\sim 1700 \mathrm{CE})$ correspond to both high- and low-rainfall intervals (cf. Fig. 11a and b). This lack of agreement arises from the strong multidecadal variability in the complete Solomons stalagmite record (1423-2010 CE) at 46, 22, and 14 years that is not present in the solar record, which contains concentrations of variance at 205, 88, and 11 years (e.g., Gray et al., 2010). On decadal-scales, we evaluate the influence of the $11 \mathrm{yr}$ solar cycle on rainfall variability in the SPCZ region, as proposed by Meehl et al. (2009), using the long stalagmite record. There is no consistent relationship between variations in sunspot number and stalagmite proxy rainfall (cf. Fig. 11a and c). There is also no consistent relationship when both records are filtered to isolate the $11 \mathrm{yr}$ component (Fig. 11d). Indeed, over the course of the 262 years of comparison, one can identify decades when the two records are in phase, out of phase, and when they have various lead-lag relations. Hence we conclude that rainfall variability at the study site is not a result of forcing related to the $11 \mathrm{yr}$ solar cycle.

We also assess the potential that volcanic forcing may influence SPCZ variability. Volcanic eruptions influence climate on multidecadal to centennial timescales, owing to the cumulative effects of multiple eruptions and accumulation of volcanic aerosols (Robock, 2000; Robock and Mao, 1995). Recent work using a fully coupled climate model (Wang et al., 2012) suggests a response in the North Pacific region of the PDV to tropical volcanism. The mechanism proposed to explain this response is a reduction of subtropical North Pacific westerlies and resulting anomalously anticyclonic winds driving the PDV in the North Pacific into a negative phase. An analogous response in the Southern Hemisphere and SPCZ region, viewed through stalagmite $\delta^{18} \mathrm{O}$ variability, would be one of isotopic depletion. However, comparison of the stalagmite $\delta^{18} \mathrm{O}$ record with a proxy record of global volcanic activity (Crowley and Unterman, 2013) shows no systematic pattern of isotopic depletion following large volcanic eruptions (e.g., $1455 \mathrm{CE}, 1809 \mathrm{CE}, 1815 \mathrm{CE}$ ) or a series of smaller eruptions (e.g., 1600-1700 CE). Indeed, there are periods of enhanced decadal variability in the stalagmite record that occur during periods of no major volcanic activity (e.g., 1456-1600 CE, 1700-1808 CE). Lacking a coherent temporal correspondence between volcanic activity and stalagmite $\delta^{18} \mathrm{O}$, we conclude that there is little relationship 


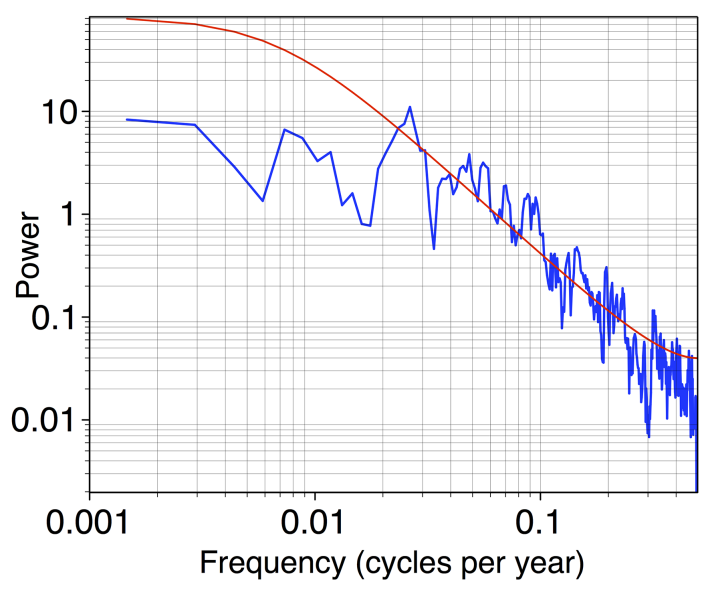

Figure 9. Multi-taper method (MTM) spectrum of the median stalagmite $\delta^{18} \mathrm{O}$ time series. The red line denotes the $99 \%$ confidence intervals for an $\mathrm{AR}(1)$ red noise model calculated from the 10FC02 stalagmite time series. The MTM analysis was performed using the K-Spectra software package (http://www.spectraworks.com/). The MTM spectrum contains significant concentrations of power throughout the interannual and multidecadal bandwidth.

between rainfall in the Solomons and volcanic activity on decadal timescales.

We also assess the potential that anthropogenic forcing may influence SPCZ variability. There is no long-term trend in the stalagmite $\delta^{18} \mathrm{O}$ record over the past 300 years, nor over the 20th century. This lack of trend contrasts with records of atmospheric $\mathrm{CO}_{2}$ and global surface temperatures. Interestingly, the dry phase of the last $\sim 30$ years in the Solomon Islands stalagmite record is not as isotopically enriched as in preceding dry phases of the PDV (Fig. 12), much like observed in the Vanuatu record (Partin et al., 2013). The relative wetness of the most recent dry phase is consistent with the hypothesized fingerprint of anthropogenic global warming on climatological regions of high rainfall amount (Held and Soden, 2006; Perkins et al., 2012). For perspective, rainfall in the tropical SPCZ region is predicted to increase by $9-20 \%$ in the next century due to anthropogenic influences (Deser et al., 2004; Perkins et al., 2012). Note that a $20 \%$ rainfall increase in the future is less than a third of the relative changes in rainfall amount that has occurred naturally on decadal timescales over the past six centuries based on our proxy-based rainfall reconstruction.

Recent modeling work (e.g., Cai et al., 2012) has demonstrated the possibility for warmer surface conditions to produce more extreme equatorward movement of the SPCZ during interannual variability, producing more interannual, El Niño-Southern Oscillation (ENSO)-related drought at our study site. Given our lack of ability to consistently resolve interannual variability in the stalagmite record, we cannot address interannual changes in the SPCZ that may be related to anthropogenic forcing.

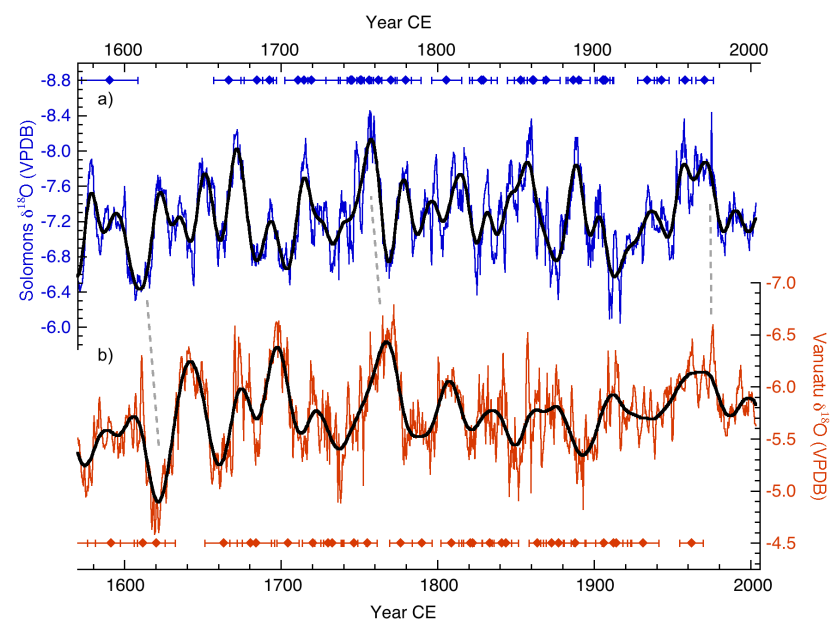

Figure 10. Comparison of stalagmite $\delta^{18} \mathrm{O}$ records from (a) Solomon Islands (blue) and (b) Vanuatu (red). Blue (red) diamonds with $\pm 2 \sigma$ error bars illustrate the locations of U-Th ages in the stalagmite from the Solomons (Vanuatu). Also plotted are the reconstructed singular spectral analysis (SSA) components (RC, thick black line) for both records over the common interval of stalagmite growth (1557-2003 CE): Solomons - the first PC (32\% variance at 48 years), the second $\mathrm{PC}$ ( $25 \%$ variance at 25 years), and the third PC (14\% variance at 14 years); Vanuatu - the first PC ( $41 \%$ variance at 56 years), the second PC (25\% variance at 30 years), and the third PC (9\% variance at 18 years). Dashed lines highlight select periods of high and low rainfall that are coeval between the sites within age model error. The two stalagmite $\delta^{18} \mathrm{O}$ records have similar variability in both the time and frequency domains, suggesting that the multidecadal variability is regional in scale. This agreement implies that changes in the SPCZ are the source of the observed variability in the stalagmite records.

In summary, although we have not performed a formal attribution analysis, we find that there is no consistent response in SPCZ rainfall on decadal to multidecadal timescales to solar, volcanic, or anthropogenic forcing. Therefore, decadalto multidecadal-scale oscillations observed in the stalagmite reconstruction most likely arise as a consequence of internal variability in the ocean-atmosphere climate system of the $\mathrm{Pa}$ cific. Such internal variability drives large changes in rainfall amount over the study site as a consequence of changes in SPCZ strength and movement. This interpretation is consistent with hypotheses suggesting that internal variability plays a major role in driving decadal-scale climate variability in the Pacific via ocean-atmosphere interactions (Clement et al., 2011; Gu and Philander, 1997; McPhaden and Zhang, 2002).

\subsection{PDV in the instrumental and stalagmite records}

The PDV contained in instrumental records can be used to assess the potential source of the multidecadal variability in the stalagmite records since 1870 , the common period amongst the records. Demonstration of a coherent relationship between instrumental PDV records and stalagmite 


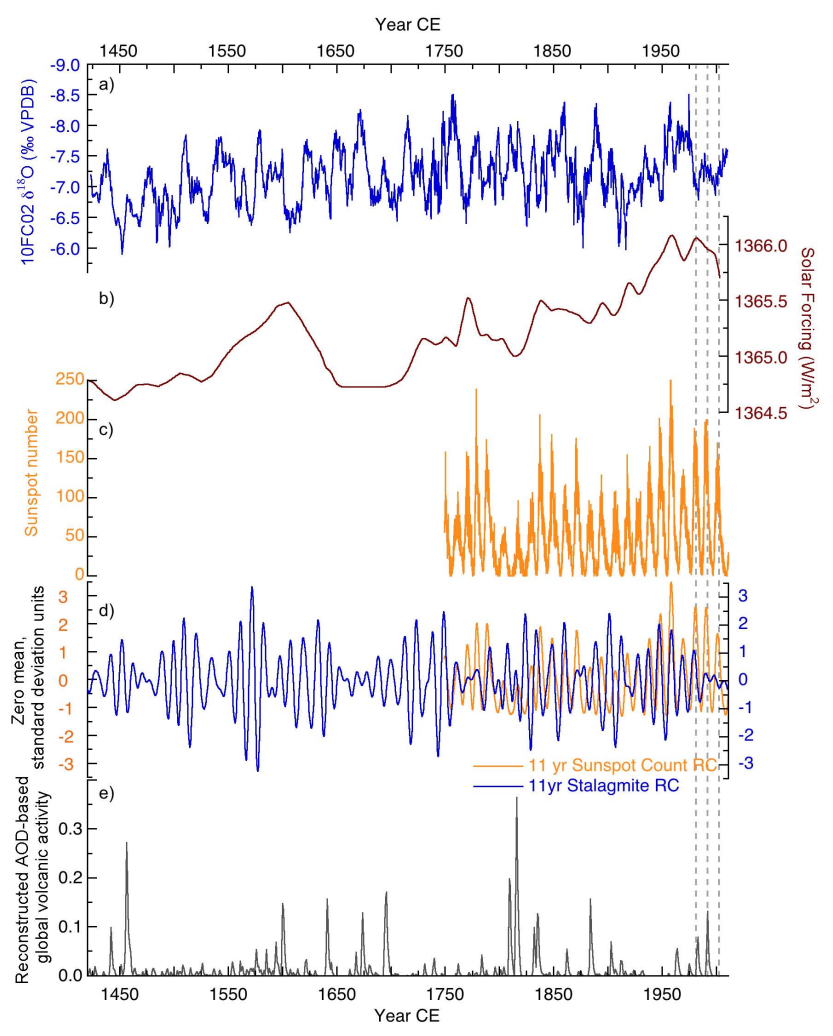

Figure 11. Stalagmite $\delta^{18} \mathrm{O}$ variations compared with records of solar and volcanic forcing over the period 1750-2009. (a) Stalagmite $\delta^{18} \mathrm{O}$ record. (b) Record of time evolution of solar forcing (Vieira et al, 2011). (c) Record of variations in sunspot number (Gray et al., 2010). (d) Reconstructed SSA components (RCs) in the stalagmite and sunspot number records, with 10 components and a 60-year window length, selected to highlight the 11-year sunspot cycle. For the stalagmite record, the fourth $\mathrm{PC}$ ( $8 \%$ variance at 12 years) and fifth PC ( $4 \%$ variance at 10 years), which average to 11 years, and account for $12 \%$ of the record variance, are used to create a stalagmite RC. For the sunspot number record, the first PC (52\% variance at 12 years) and second PC (33\% variance at 10 years), again averaging to 11 years, are used to create a sunspot RC. The most recent three peaks of sunspot activity used by Matthews (2011) and Meehl et al. (2009) to hypothesize a response in SPCZ precipitation to changing sunspot numbers are marked by vertical dashed lines. (e) illustrates the volcanic aerosol optical depth (AOD) proxy reconstruction of global volcanic activity from Crowley and Unterman (2012). There is no systematic relationship in phasing or amplitude modulation of the stalagmite record that supports external forcing by solar or volcanoes as a driver of multidecadal SPCZ variability.

proxy rainfall reconstructions increases the confidence in the stalagmite-based reconstructions of PDV. The timing of recent decadal shifts in SPCZ rainfall documented by the two stalagmite records is broadly consistent with the timing of phase changes (wet phases: 1900-1924, 1947-1976; dry phases: 1925-1946, 1977-1995) in other relevant tropical Pacific instrumental time series (Fig. 12): the IPO index of SST, equatorial central Pacific SST, as well as the zonal

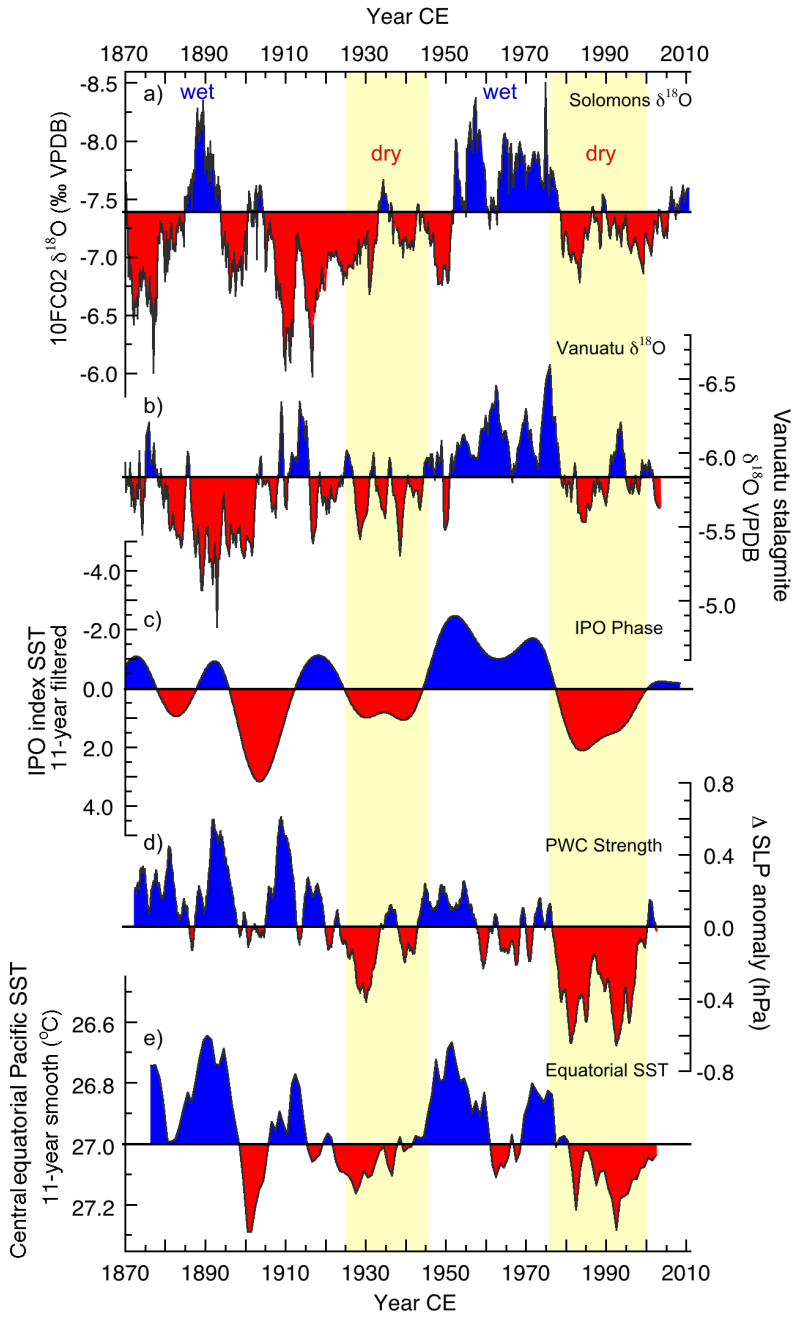

Figure 12. PDV variability as manifest in stalagmite $\delta^{18} \mathrm{O}$ and select ocean and atmospheric records since 1870 CE. (a) Solomons stalagmite $\delta^{18} \mathrm{O}$, (b) Vanuatu stalagmite $\delta^{18} \mathrm{O}$, (c) Interdecadal Pacific Oscillation index (IPO) of SST anomalies (Folland et al., 2002), (d) sea level pressure (SLP) gradient anomaly as calculated by Vecchi et al. (2006) from the Kaplan SLP data set, taken in the literature as a surface expression of Pacific Walker circulation (PWC) strength, and (e) 11-year running average of annual mean central tropical Pacific SST over $5^{\circ} \mathrm{S}$ to $5^{\circ} \mathrm{N}, 120^{\circ} \mathrm{W}$ to $170^{\circ} \mathrm{W}$. Similar smoothing has been used in previous examinations of decadal SST variability in the central Pacific (e.g. Zhang et al. 2006). Identified phases occurred from 1925 through 1946 (22 yr duration), 1947 through 1976 (30 yr duration), and 1977 through 1998 (22 yr duration). There is better agreement between the proxy records and the climate records in the more recent times than in earlier times. This lack of agreement might be caused by decreases in the signal-tonoise ratio in the earliest portion of the instrumental records (e.g., Giese and Ray, 2011; L'Heureux et al., 2013) and age model uncertainties in the stalagmite records. This suggests that rainfall, sea level pressure, and SST change in step on multidecadal timescales, and are also associated with basin-wide reorganizations associated with PDV. 
Pacific SLP gradient, where reliable SLP data are available ( $~ 1950$ to present; L'Heureux et al., 2013). The SLP gradient in the tropical Pacific has been taken as a surface expression of the PWC (Deser et al., 2004; Dong and Lu, 2013; Vecchi and Soden, 2007; Vecchi et al., 2006). Post-1950, when the quality of the instrumental records is best, there is good agreement between the proxy and instrumental records, as well as between the individual instrumental records themselves. All records capture the wet phase from 1947 to 1976 and dry phase from 1977 to 1995 , as well as the timing of the transition in 1976/1977. Although the quality of instrumental data decreases pre-1950, all records capture the dry phase from 1925 to 1946, as well as the transition in 1946/1947. Pre-1925, the records do not agree on the timing, duration, and even phase of the PDV. This lack of agreement might be caused by decreases in the signal-to-noise ratio in the earliest portion of the instrumental records and age model uncertainties in the stalagmite records.

We look in more detail at the well-documented climate regime shift of 1976/1977 to investigate the processes behind the observed phase changes in instrumental and proxy records. Reduced surface trade winds and easterlies aloft, and a weakening in the thermally direct Walker circulation, mean less latent heat flux into the atmosphere by trade-winddriven evaporative cooling. This reduced trade wind strength has been documented by Folland et al. (2002) to allow a northeastward displacement of the SPCZ. It stands to reason that such a response mechanism to ocean-atmosphere PDV would be in operation prior to the 1976/1977 shift. Additionally, from a thermodynamic standpoint, a reduced latent heat flux into the atmosphere means that less rainfall in the tropical SPCZ and WPWP deep convection regions is required to satisfy the radiation budget.

\section{Summary and conclusions}

In this study, we have presented a new, U-series-dated stalagmite $\delta^{18} \mathrm{O}$ record for the past six centuries. A relationship is developed between $\delta^{18} \mathrm{O}$ variations in a fast-growing stalagmite and local rainfall amount to produce a $600 \mathrm{yr}$ record of rainfall variability from the tropical portion of the SPCZ. We present evidence for large $(\sim 1.5 \mathrm{~m})$, abrupt, and periodic changes in total annual rainfall amount on decadal to multidecadal timescales since $1423 \pm 5 \mathrm{CE}$ in the Solomon Islands. The pre-instrumental portion of the stalagmite $\delta^{18} \mathrm{O}$ record contains decadal transitions that routinely reach or exceed 1.5 mTAR $(\sim 2.4 \%$ o). Such values are three times as large as the wet-to-dry transition of $0.5 \mathrm{mTAR}(\sim 0.6 \%)$ that occurred in 1976/1977.

A comparison between the stalagmite $\delta^{18} \mathrm{O}$ records at the Solomon Islands and Vanuatu establishes the regional coherence of the reconstructed rainfall variability associated with the SPCZ. Spectral analysis reveals that both stalagmite records share $>70 \%$ of their variance at periods between 16 and 52 years. The timing of the decadal changes in rainfall inferred from the 20th-century portion of the stalagmite $\delta^{18} \mathrm{O}$ records coincides with previously identified decadal shifts in Pacific ocean-atmosphere behavior (Clement et al., 2011; Deser et al., 2004). We find no coherent temporal correspondence on decadal to multidecadal timescales between external forcing (solar, volcanic, or anthropogenic) and stalagmite $\delta^{18} \mathrm{O}$ variations. Rather, multidecadal rainfall variability in the SPCZ appears to be a consequence of internal variability in the ocean-atmosphere climate system.

The $600 \mathrm{yr}$ Solomon Islands stalagmite $\delta^{18} \mathrm{O}$ record indicates that decadal oscillations in rainfall are a persistent characteristic of SPCZ-related climate variability, which is important for testing climate model skill as well as informing future projections. Furthermore, future occurrences of such large, decades-long changes in rainfall within the SPCZ and WPWP deserve careful consideration when arriving at strategies for infrastructure development and resource management in the SPCZ region as well as the many regions that are teleconnected to Pacific decadal variability (Deser et al., 2004; Garreaud and Battisti, 1999; L'Heureux et al., 2013; Zhang et al., 1997).

Acknowledgements. The authors would like to acknowledge Douglas Billy, Director of the Geological Survey Division, and Alison Papabatu, Head of the Seismology Section of The Solomon Islands Geological Survey, Division of the Ministry of Mines, Energy and Rural Electrification, for graciously providing permits that made this work possible. We would like to thank Don Austin and Jimmy of Honiara for their invaluable assistance with enabling transportation in the field. Chief Joseph and the locals of Barana Village on Mount Austen helped both to locate and allowed access to Forestry Cave and generously gave permission to collect dripwater and stalagmite samples. We also acknowledge thoughtful comments, along with helpful discussions and contributions, from Kevin Anchukaitis, Meaghan Gorman, Kelly Hereid, Julien EmileGeay, Jessica Tierney, Kristine DeLong, and Deborah Khider. This study was supported by Taiwan ROC NSC and NTU grants (101-2116-M-002-009, 102-2116-M-002-016, and 101R7625 to CCS).

Edited by: P. Braconnot

\section{References}

Cai, W. W., Lengaigne, M. M., Borlace, S. S., Collins, M. M., Cowan, T. T., McPhaden, M. J. M., Timmermann, A. A., Power, S. S., Brown, J. J., Menkes, C. C., Ngari, A. A., Vincent, E. M. E., and Widlansky, M. J. M.: More extreme swings of the South Pacific convergence zone due to greenhouse warming, Nature, 488, 365-369, doi:10.1038/nature11358, 2012.

Cane, M. A.: Climate science: decadal predictions in demand, Nat. Geosci., 3, 231-232, doi:10.1038/ngeo823, 2010. 
Cheng, H., Edwards, R. L., Hoff, J., Gallup, C. D., Richards, D. A., and Asmerom, Y.: The half-lives of uranium-234 and thorium230, Chem. Geol., 169, 17-33, 2000.

Clement, A., DiNezio, P., and Deser, C.: Rethinking the Ocean's Role in the Southern Oscillation, J. Climate, 24, 4056-4072, doi:10.1175/2011JCLI3973.1, 2011.

Cravatte, S., Delcroix, T., Zhang, D., Mcphaden, M., and Leloup, J.: Observed freshening and warming of the western Pacific Warm Pool, Clim. Dynam., 33, 565-589, doi:10.1007/s00382-0090526-7, 2009.

Crowley, T. J.: Causes of Climate Change Over the Past 1000 Years, Science, 289(5477), 270-277, doi:10.1126/science.289.5477.270, 2000.

Crowley, T. J. and Unterman, M. B.: Technical details concerning development of a $1200 \mathrm{yr}$ proxy index for global volcanism, Earth Syst. Sci. Data, 5, 187-197, doi:10.5194/essd-5-187-2013, 2013.

Dansgaard, W.: Stable isotopes in precipitation, Tellus, 16, 436468, 1964

Deser, C., Phillips, A., and Hurrell, J.: Pacific interdecadal climate variability: Linkages between the tropics and the North Pacific during boreal winter since 1900, J. Climate, 17, 3109-3124, 2004.

Di Lorenzo, E., Cobb, K., Furtado, J., Schneider, N., Anderson, B., Bracco, A., Alexander, M., and Vimont, D.: Central Pacific El Nino and decadal climate change in the North Pacific Ocean, Nat. Geosci., 3, 762-765, doi:10.1038/NGEO984, 2010.

Dong, B. and $\mathrm{Lu}, \mathrm{R}$.: Interdecadal enhancement of the walker circulation over the Tropical Pacific in the late 1990s, Adv. Atmos. Sci., 30, 247-262, doi:10.1007/s00376-012-2069-9, 2013.

Dorale, J. A. and Liu, Z.: Limitations of Hendy test criteria in judging the paleoclimatic suitability of speleothems and the need for replication, J. Cave Karst Stud., 71, 73-80, 2009.

Feng, W., Banner, J. L., Guilfoyle, A. L., Musgrove, M., and James, E. W.: Oxygen isotopic fractionation between drip water and speleothem calcite: a $10 \mathrm{yr}$ monitoring study, Central Texas, USA, Chem. Geol., 304-305, 53-67, doi:10.1016/j.chemgeo.2012.02.004, 2012.

Folland, C., Renwick, J., Salinger, M., and Mullan, A.: Relative influences of the Interdecadal Pacific Oscillation and ENSO on the South Pacific Convergence Zone, Geophys. Res. Lett., 29, 1643, doi:10.1029/2001GL014201, 2002.

Garreaud, R. D. and Battisti, D. S.: Interannual (ENSO) and interdecadal (ENSO-like) variability in the Southern Hemisphere tropospheric circulation, J. Climate, 12, 2113-2123, 1999.

Gat, J. R.: Oxygen and hydrogen isotopes in the hydrologic cycle, Annu. Rev. Earth. Planet. Sc., 24, 225-262, 1996.

Goddard, L., Hurrell, J. W., Kirtman, B. P., Murphy, J., Stockdale, T., and Vera, C.: Two time scales for the price of one (almost), B. Am. Meteorol. Soc., 93, 621-629, doi:10.1175/BAMSD-11-00220.1, 2012.

Gorman, M. K., Quinn, T. M., Taylor, F. W., Partin, J. W., Cabioch, G., Austin, J. A., Jr., Pelletier, B., Ballu, V., Maes, C., and Saustrup, S.: A coral-based reconstruction of sea surface salinity at Sabine Bank, Vanuatu from 1842 to 2007 CE, Paleoceanography, 27, PA3226, doi:10.1029/2012PA002302, 2012.

Gray, L. J., Beer, J., Geller, M., Haigh, J. D., Lockwood, M., Matthes, K., Cubasch, U., Fleitmann, D., Harrison, G., Hood, L., Luterbacher, J., Meehl, G. A., Shindell, D., Van Geel, B., and White, W.: Solar Influences on Climate, Rev. Geophys., 48, RG4001, doi:10.1029/2009RG000282, 2010.

$\mathrm{Gu}, \mathrm{D}$. and Philander, S.: Interdecadal climate fluctuations that depend on exchanges between the tropics and extratropics, Science, 275, 805-807, 1997.

Held, I. M. and Soden, B. J.: Robust responses of the hydrological cycle to global warming, J. Climate, 19, 5686-5699, 2006.

Hendy, C. H.: Isotopic geochemistry of speleothems: 1. calculation of effects of different modes of formation on isotopic composition of speleothems and their applicability as palaeoclimatic indicators, Geochim. Cosmochim. Ac., 35, 801-824, doi:10.1016/0016-7037(71)90127-X, 1971.

Jaffey, A. H., Flynn, K. F., Glendenin, L. E., Bentley, W. C., and Essling, A. M.: Precision measurement of half-lives and specific activities of U-235 and U-238, Phys. Rev. C, 4, 1889-1906, 1971.

Kim, S.-T. and O'Neil, J. R.: Equilibrium and nonequilibrium oxygen isotope effects in synthetic carbonates, Geochim. Cosmochim. Ac., 61, 3461-3475, 1997.

Kummerow, C., Simpson, J., Thiele, O., Barnes, W., Chang, A., Stocker, E., Adler, R. F., Hou, A., Kakar, R., Wentz, F., Ashcroft, P., Kozu, T., Hong, Y., Okamoto, K., Iguchi, T., Kuroiwa, H., Im, E., Haddad, Z., Huffman, G., Ferrier, B., Olson, W. S., Zipser, E., Smith, E. A., Wilheit, T. T., North, G., Krishnamurti, T., and Nakamura, K.: The status of the Tropical Rainfall Measuring Mission (TRMM) after two years in orbit, J. Appl. Meteorol., 39, 1965-1982, 2000.

Kurita, N.: Water isotopic variability in response to mesoscale convective system over the tropical ocean, J. Geophys Res.-Atmos, 118, 10376-10390, doi:10.1002/jgrd.50754, 2013.

Liu, Y., Liang, X., and Weisberg, R. H.: Rectification of the bias in the wavelet power spectrum, J. Atmos. Oceanic Technol., 24, 2093-2102, doi:10.1175/2007JTECHO511.1, 2007.

L'Heureux, M. L., Lee, S., and Lyon, B.: Recent multidecadal strengthening of the Walker circulation across the Tropical Pacific, Nature Clim. Change, doi:10.1038/nclimate1840, 2013.

Mantua, N. J. and Hare, S. R.: The Pacific decadal oscillation, J. Oceanogr., 58, 35-44, 2002.

Mantua, N. J., Hare, S. R., Zhang, Y., Wallace, J. M., and Francis, R. C.: A Pacific interdecadal climate oscillation with impacts on salmon production, B. Am. Meteorol. Soc., 78, 1069-1079, 1997.

Matthews, A. J.: A multiscale framework for the origin and variability of the South Pacific Convergence Zone, Q. J. Roy. Meteor. Soc., 1165-1178, doi:10.1002/qj.1870, 2011.

McPhaden, M. and Zhang, D.: Slowdown of the meridional overturning circulation in the upper Pacific Ocean, Nature, 415, 603608, 2002.

Meehl, G. A., Arblaster, J. M., Matthes, K., Sassi, F., and van Loon, H.: Amplifying the Pacific climate system response to a small $11 \mathrm{yr}$ solar cycle forcing, Science, 325, 1114-1118, doi:10.1126/science.1172872, 2009.

Mickler, P. J., Stern, L. A. and Banner, J. L.: Large kinetic isotope effects in modern speleothems, Geol. Soc. Am. Bull., 118, 6581, doi:10.1130/B25698.1, 2006.

Newman, M., Compo, G. P., and Alexander, M. A.: ENSO-forced variability of the Pacific decadal oscillation, J. Climate, 16, 3853-3857, 2003.

Partin, J. W., Jenson, J. W., Banner, J. L., Quinn, T. M., Taylor, F. W., Sinclair, D., Hardt, B., Lander, M. A., Bell, T., 
Miklaviè, B., Jocson, J. M. U., and Taboroši, D.: Relationship between modern rainfall variability, cave dripwater, and stalagmite geochemistry in Guam, USA, Geochem. Geophy. Geosy., 13, Q03013, doi:10.1029/2011GC003930, 2012.

Partin, J. W., Quinn, T. M., Shen, C. C., Emile-Geay, J., Taylor, F. W., Maupin, C. R., Lin, K., Jackson, C. S., Banner, J. L. and Sinclair, D. J.: Multidecadal rainfall variability in South Pacific Convergence Zone as revealed by stalagmite geochemistry, Geol, 41(11), 1143-1146, 2013.

Perkins, S., Irving, D., Brown, J., Power, S., Moise, A., Colman, R., and Smith, I.: CMIP3 ensemble climate projections over the -western tropical Pacific based on model skill, Clim. Res., 51, 35-58, doi:10.3354/cr01046, 2012.

Rayner, N., Parker, D. E., Horton, E., Folland, C., Alexander, L., Rowell, D., Kent, E., and Kaplan, A.: Global analyses of sea surface temperature, sea ice, and night marine air temperature since the late nineteenth century, J. Geophys. Res.-Atmos., 108, 4407, doi:10.1029/2002JD002670, 2003.

Robock, A.: Volcanic eruptions and climate, Rev. Geophys., 38, 191-219, 2000.

Robock, A. and Mao, J.: The volcanic signal in surface temperature observations, J. Climate, 8, 1086-1103, 1995.

Rozanski, K., Araguas-Araguas, L., and Gonfiantini, R.: Isotopic patterns in modern global precipitation, in: Climate Change in Continental Isotopic Records, American Geophysical Union, Washington, DC, 1-36, 1993.

Shen, C.-C., Wu, C.-C., Cheng, H., Edwards, R. L., Hsieh, Y.T., Gallet, S., Chang, C.-C., Li, T.-Y., Lam, D. D., Kano, A., Hori, M., and Spötl, C.: High-precision and high-resolution carbonate ${ }^{230} \mathrm{Th}$ dating by MC-ICP-MS with SEM protocols, Geochim. Cosmochim. Ac., 99, 71-86, doi:10.1016/j.gca.2012.09.018, 2012.

Takahashi, K. and Battisti, D. S.: Processes controlling the mean tropical pacific precipitation pattern. Part II: The SPCZ and the southeast pacific dry zone, J. Climate, 20, 5696-5706, doi:10.1175/2007JCLI1656.1, 2007.

Thirumalai, K., Singh, A., and Ramesh, R.: A MATLAB Code to Perform Weighted Linear Regression with (correlated or uncorrelated) Errors in Bivariate Data, J. Geol. Soc. India, 77, 377380, 2011.

Torrence, C. and Compo, G. P.: A practical guide to wavelet analysis, B. Am. Meteorol. Soc., 79, 61-78, 1998.
Tremaine, D. M., Froelich, P. N., and Wang, Y.: Speleothem calcite farmed in situ: Modern calibration of delta O-18 and delta C-13 paleoclimate proxies in a continuously-monitored natural cave system, Geochim. Cosmochim. Ac., 75, 4929-4950, doi:10.1016/j.gca.2011.06.005, 2011.

Vecchi, G. A. and Soden, B. J.: Global warming and the weakening of the tropical circulation, J. Climate, 20, 4316-4340, doi:10.1175/JCLI4258.1, 2007.

Vecchi, G., Soden, B., Wittenberg, A., Held, I., Leetmaa, A., and Harrison, M.: Weakening of tropical Pacific atmospheric circulation due to anthropogenic forcing, Nature, 441, 73-76, doi:10.1038/nature04744, 2006.

Vieira, L. E. A., Solanki, S. K., Krivova, N. A. and Usoskin, I.: Evolution of the solar irradiance during the Holocene, A\&A, 531, A6, doi:10.1051/0004-6361/201015843, 2011.

Vincent, D. G.: The South-Pacific Convergence Zone (SPCZ) - a review, Mon. Weather Rev., 122, 1949-1970, 1994.

Vose, R. S., Schmoyer, R. L., Steurer, P. M., Peterson, T. C., Heim, R., Karl, T. R., and Eischeid, J. K.: The Global Historical Climatology Network: long-term monthly temperature, precipitation, sea level pressure, and station pressure data, Oak Ridge National Laboratory, ORNL/CDIAC-53(NPD-041), doi:10.3334/CDIAC/cli.ndp041, 1992.

Wang, T., Otterå, O. H., Gao, Y., and Wang, H.: The response of the North Pacific Decadal Variability to strong tropical volcanic eruptions, Clim. Dyn., 39, 2917-2936, 2012.

Widlansky, M. J., Webster, P. J., and Hoyos, C. D.: On the location and orientation of the South Pacific Convergence Zone, Clim. Dynam., 36, 561-578, doi:10.1007/s00382-010-0871-6, 2010.

Zhang, D. and McPhaden, M. J.: Decadal variability of the shallow Pacific meridional overturning circulation: Relation to tropical sea surface temperatures in observations and climate change models, Ocean Model., 15, 250-273, doi:10.1016/j.ocemod.2005.12.005, 2006.

Zhang, Y., Wallace, J. M., and Battisti, D. S.: ENSO-like interdecadal variability: 1900-93, J. Climate, 10, 1004-1020, 1997. 